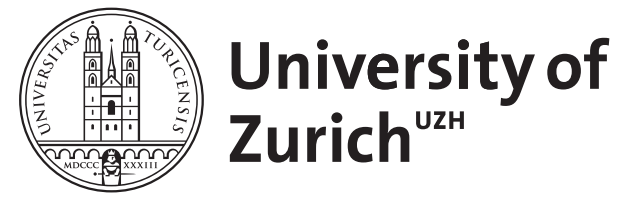

\title{
Diabetes und Stoffwechsel
}

von Eckardstein, Arnold ; Minder, Elisabeth

DOI: https://doi.org/10.1515/9783110228731.80

Posted at the Zurich Open Repository and Archive, University of Zurich

ZORA URL: https://doi.org/10.5167/uzh-74345

Book Section

Published Version

Originally published at:

von Eckardstein, Arnold; Minder, Elisabeth (2012). Diabetes und Stoffwechsel. In: Hoffmann, W; Aufenanger, J; Hoffmann, G. Klinikhandbuch Labordiagnostische Pfade. Berlin / Boston: Walter de Gruyter, 80-103.

DOI: https://doi.org/10.1515/9783110228731.80 
Wada N, Kubo M, Kijima H, Ishizuka T, Saeki T, Koike T, Sasano H. 1996. Adrenocorticotropin-independent bilateral macronodular adrenocortical hyperplasia: immunohistochemical studies of steroidogenic enzymes and post-operative course in two men. Eur J Endocrinol 134(5): 583-7.

Wajchenberg BL, Mendonca BB, Liberman B, Pereira MA, Carneiro PC, Wakamatsu A, Kirschner MA. 1994. Ectopic adrenocorticotropic hormone syndrome. Endocr Rev 15(6): 752-87.

Wigg SJ, Ehrlich AR, Fuller PJ. 1999. Cushing's syndrome secondary to ectopic ACTH secretion from metastatic breast carcinoma. Clin Endocrinol (Oxf) 50(5): 675-8.

Williams GH, Dluhy RG. 2008. Disorders of the Adrenal Cortex. In: Fauci AS, Braunwald E, Kasper DL, Hauser SL, Longo DL, Jameson JL, Loscalzo J, editors. Harrison's Principles of Internal Medicine: McGraw-Hill. p 2247-2268.

Wood JB, Frankland AW, James VH, Landon J. 1965. A Rapid Test of Adrenocortical Function. Lancet 1 (7379): 243-5.

Yanovski JA, Cutler GB, Jr., Chrousos GP, Nieman LK. 1993. Corticotropin-releasing hormone stimulation following low-dose dexamethasone administration. A new test to distinguish Cushing's syndrome from pseudo-Cushing's states. JAMA 269(17): 2232-8.

Zarate A, Kovacs K, Flores M, Moran C, Felix I. 1986. ACTH and CRF-producing bronchial carcinoid associated with Cushing's syndrome. Clin Endocrinol (Oxf) 24(5): 523-9.

Zeiger MA, Nieman LK, Cutler GB, Chrousos GP, Doppman JL, Travis WD, Norton JA. 1991. Primary bilateral adrenocortical causes of Cushing's syndrome. Surgery 110(6): 1106-15.

\subsection{Diabetes und Stoffwechsel}

\section{Arnold von Eckardstein, Elisabeth Minder}

\subsubsection{Diabetes mellitus}

\subsubsection{Diagnostik und Risikoabschätzung}

1979/1980 legten die National Diabetes Data Group und die Weltgesundheitsorganisation (WHO) die diagnostischen Kriterien für Diabetes mellitus erstmals aufgrund epidemiologischer Daten fest, nämlich 1. das Vorhandensein klassischer Symptome bei Nachweis einer Hyperglykämie, 2. Glukosekonzentrationen $>7.8 \mathrm{mmol} / \mathrm{L}(>140$ $\mathrm{mg} / \mathrm{dl}$ ) im Nüchternplasma (fasting plasma glucose $=\mathrm{FPG})$, 3. eine Glukosekonzentrationen von $>11.1 \mathrm{mmol} / \mathrm{L}$ (> $200 \mathrm{mg} / \mathrm{dL}) 2 \mathrm{~h}$ nach oraler Glukosebelastung (2h-PG nach OGTT) (National Diabetes Group 1979). Eine $2 \mathrm{hPG}$ im OGTT wurde
Referenzgrösse, weil dieser Cut-Off in den damals analysierten Querschnittsanalysen von epidemiologischen Daten am besten mit dem Auftreten einer Retinopathie korrelierte. Diese Definitionen wurden Ende der 1990er Jahre aufgrund zahlreicher neuer prospektiver epidemiologischer Studienergebnisse von der American Diabetic Association (ADA) und der WHO revidiert (American Diabetes Association 1997; World Health Organisation 1999). Der diagnostische Cut-Off für FPG wurde auf 7,0 mmol/L (126 mg/dL) abgesenkt, weil das Überschreiten diese Grenzwertes am sensitivsten das Überschreiten der 2h-PG von $11,1 \mathrm{mmol} / \mathrm{L}(200 \mathrm{mg} / \mathrm{dL})$ im OGTT voraussagt. Zusätzlich wurde von der ADA der Nachweis einer zufällig, also unabhängig vom prandialen Zustand, $>11,1 \mathrm{mmol} / \mathrm{L}$ erhöhten Glukose-Plasmakonzentration als diagnostisches Kriterium aufgenommen, während die WHO die OGTT-Kriterien beibehielt. Außerdem wurde von der ADA die impaired fasting glucose (IFG: $6,1-7,0 \mathrm{mmol} / \mathrm{L}=$ 110-126 mg/dL, später revidiert auf 5,6-7,0 mmol/L $=100-126 \mathrm{mg} / \mathrm{dL}$ ) als prognostisches Kriterium für die Feststellung eines erhöhten Risikos für die spätere Entwicklung eines Diabetes mellitus aufgenommen (American Diabetes Association 1997, 2010) während die WHO an der Definition der impaired glucose tolerance (IGT) durch eine $2 \mathrm{~h}$ PG von 7,8-11,1 mmol/L (140-200 mg/dL) im OGTT festhielt (World Health Organisation 1999).

Anfangs 2010 publizierte die ADA eine abermalige Revision der Kriterien für die Diagnostik und Klassifikation des Diabetes mellitus (American Diabetes Association 2010): Die neuen Empfehlungen fassen die früheren ADA- und WHO-Definitionen für die Diagnose des Diabetes mellitus zusammen und nehmen neu die ein Jahr zuvor von einer internationalen Expertengruppe vorgeschlagenen Cut-Offs für glykiertes Hämoglobin A1 (HbA1c) (International Expert Committee 2009) zur Diagnose eines manifesten Diabetes mellitus auf. Die Definitionen sind in der $\triangleright$ Tabelle $4.7 \mathrm{zu}$ sammengefasst. Der Begriff des Prädiabetes wurde ersetzt durch „Kategorie mit erhöhtem Risiko für einen Diabetes mellitus" ( $\triangleright$ Tab. 4.8).

Hauptargumente für die Aufnahme der HbA1cKonzentration in die Diagnosekriterien des Diabetes mellitus sind die Unzuverlässigkeit der Plasmaglukose-Konzentration aufgrund diurnaler und prandialer Schwankungen aber auch akutem Stress, der durch Glykolyse bewirkten Instabilität der Glukose in der gewonnenen Blutprobe sowie der ana- 
Tab. 4.7 Definition des Diabetes mellitus nach den Kriterien der American Diabetes Association (2010).

\author{
Kriterien \\ 1. $\quad \mathrm{HbA} 1 \mathrm{c} \geq 6.5 \%$ nach $\mathrm{NGSP}(>48 \mathrm{mmol} / \mathrm{mol}$ nach IFCC) * \\ oder \\ 2. Nüchtern-Plasmaglukose (FPG) $\geq 126 \mathrm{mg} / \mathrm{dl}(7.0 \mathrm{mmol} / \mathrm{lL})$. Fasten ist definiert als keine kalorische Auf- \\ nahme für mindestens 8 h.*
}

oder

3. 2-Stunden Plasmaglukose $\geq 11.1 \mathrm{mmol} / \mathrm{L}$ (> $200 \mathrm{mg} / \mathrm{dL}$ ) während eines OGTT. Der Test soll nach dem Protokoll der WHO durchgeführt werden, also durchTrinken einer wässrigen Lösung mit $75 \mathrm{~g}$ unhydrierter Glukose.*

oder

4. Patient mit klassischen Symptomen der Hyperglyämie oder hyperglykämischer Krise und einer vom prandialen Zustand unabhängigen Plasmaglukose $\geq 11.1 \mathrm{mmol} / \mathrm{L}$ (> $200 \mathrm{mg} / \mathrm{dL}$ ).

\footnotetext{
* Bei nicht ausgeprägter Hyperglykämie sollten die Kriterien 1-3 durch Wiederholungsuntersuchungen bestätigt werden. FPG = fasting plasma glucose, NGSP = National Glycohemoglobin Standardization Programme, IFCC = International Federation of Clinical Chemistry
}

Tab. 4.8 Definition des Prädiabetes mellitus („Kategorie mit erhöhtem Risiko für einen Diabetes mellitus") nach den Kriterien der American Diabetes Association (2010).

\section{Kriterien*}

- FPG 5,6-6,9 mmol/l (100-125 mg/dL) (IFG)

- 2-h PG nach 75-g OGTT 7,8-11,0 mmol/L (140-199 mg/dL) (IGT)

- HbA1c 5,7-6,4\% nach NGSP (39-48 mmol/mol nach IFCC)

* Für alle 3 Tests ist das Risiko kontinuierlich, d. h., es setzt sich auch unterhalb des unteren Grenzwertes fort und nimmt am oberen Ende des Ranges überproportional zu. FPG $=$ Nüchtern-Plasmaglukose, IFG = impaired fasting glucose = erhöhte Glukosekonzentration im Nüchternplasma; 2h-PG: 2-Stunden Plasmaglukose, IGT = impaired glucose tolerance $=$ gestörte Glukosetoleranz

lytischen Interferenzen durch Medikamente (International Expert Committee 2009). Die HbA1cKonzentration ist ein intraindividuell stabilerer Biomarker, dessen Konzentration die Glykämie der vergangenen 8-12 Wochen reflektiert und dessen Präanalytik einfacher beherrschbar als die der Glukose ist. Die HbA1c-Konzentration korreliert über den gesamten Konzentrationsbereich mit dem Risiko mikrovaskulärer Komplikationen. Heute ist durch weltweite Standardisierung nach NGSP/ DCCT oder IFCC die Methodenunabhängigkeit von HbA1c-Messungen soweit optimiert, dass der Parameter für die Diagnostik in breiten Populationen eingesetzt werden kann. Wichtige bekannte Nachteile des $\mathrm{HbA1c}$ sind die im Vergleich zur Glukosemessung höhere Impräzision, insbesondere bei Verwendung von POCT-Geräten (Lenters-Westra and Slingerland 2010), sowie die möglichen Fehlbeurteilungen bei Patienten mit veränderter Erythrozytenüberlebenszeit, chronischer Niereninsuffizienz, schwerem Alkoholkonsum, oder genetischen Hämoglobin- oder Hyperglykierungsvarianten. Zudem ist der HbA1c-Test erheblich teurer als die Glukosemessung.

Ergebnisse aus populationsweiten Evaluationsstudien in Amerika, Europa und Asien weisen auf eine im Vergleich zu den Glukose-Kriterien geringere Sensitivität der HbA1c-Kriterien für die Diagnose des manifesten Diabetes mellitus. In Deutschland zeigte die Validierung der HbA1cKriterien bei 2660 31-75 Jahre alten Teilnehmern der KORA-Studie ohne bekannten Diabetes mellitus (Rathmann et al. 2011), dass die HbA1c- und Glukose-Kriterien (d.h. FPG und OGTT) unterschiedliche Personen mit Diabetes mellitus diagnostizieren: Nur $20 \%$ aller Personen, die mindestens eins der beiden Diagnosekriterien für Diabetes mellitus erfüllten, hatten ein erhöhtes HbA1c und einen pathologischen OGTT. Unter den neudiagnostizierten Diabetikern fanden sich bei nur $0,7 \%$ der 31-60-Jährigen und 2,9\% der 61-75-Jährigen ein $\mathrm{HbA} 1 \mathrm{c}>6,5 \%$ (> $48 \mathrm{mmol} /$ mol), aber bei $2,3 \%$ bzw. $7,9 \%$ pathologische OGTT-Ergebnisse. Insgesamt scheinen somit auch in Deutschland die amerikanischen HbA1c-Krite- 
rien weniger sensititv als die Glukose-Kriterien für die Diagnosen von sowohl manifestem als auch latentem Diabetes mellitus. Aus diesen Gründen haben Experten der Deutschen Diabetes-Gesellschaft (DDG) vorgeschlagen, die ADA-Empfehlungen in modifizierter Form zu übernehmen $($ Abb. 4.5) (Kerner et al. 2010)

(http://www.deutsche-diabetes-gesellschaft.de/ redaktion/news/Stellungnahme_HbA1c_final.pdf).

1. Der wichtigste Unterschied ist die Differenzierung von unterschiedlichen Cut-Offs für den Einschluss (HbA1c > 6,5\% bzw. $>48 \mathrm{mmol} /$ mol) und Ausscluss (HbA1c $<5,7 \%$ bzw. $<39 \mathrm{mmol} / \mathrm{mol}$ ) des Diabetes mellitus.
2. Im Graubereich entsprechend der "ADA-Kategorie mit erhöhtem Risiko für Diabetes mellitus" $(5,7 \%<\mathrm{HbA} 1 \mathrm{c}<6,5 \%$ bzw. $39 \mathrm{mmol} /$ $\mathrm{mol}<\mathrm{HbA} 1 \mathrm{c}<48 \mathrm{mmol} / \mathrm{mol}$ ) sollen zur weiteren Abklärung FPG und 2h-PG nach OGTT gemessen werden

3. Die Verwendung der HbA1c-Kriterien erfordert die Verwendung von NGSP/DCCT und/oder IFCC-standardisierten Methoden

4. HbA1c-Messungen sollen bei allen Personen durchgeführt werden, welche laut Deutschen Diabetes Risikotest ein erhöhtes Diabetes-Risiko haben ( $\triangleright$ Tab. 4.9) (Schulze et al. 2007) (http://www.dife.de/de/presse/Diabetes_Test_ Fragebogen.pdf)

\section{Empfehlungen der Deutschen Diabetes Gesellschaft zur Diabetes mellitus Diagnostik}

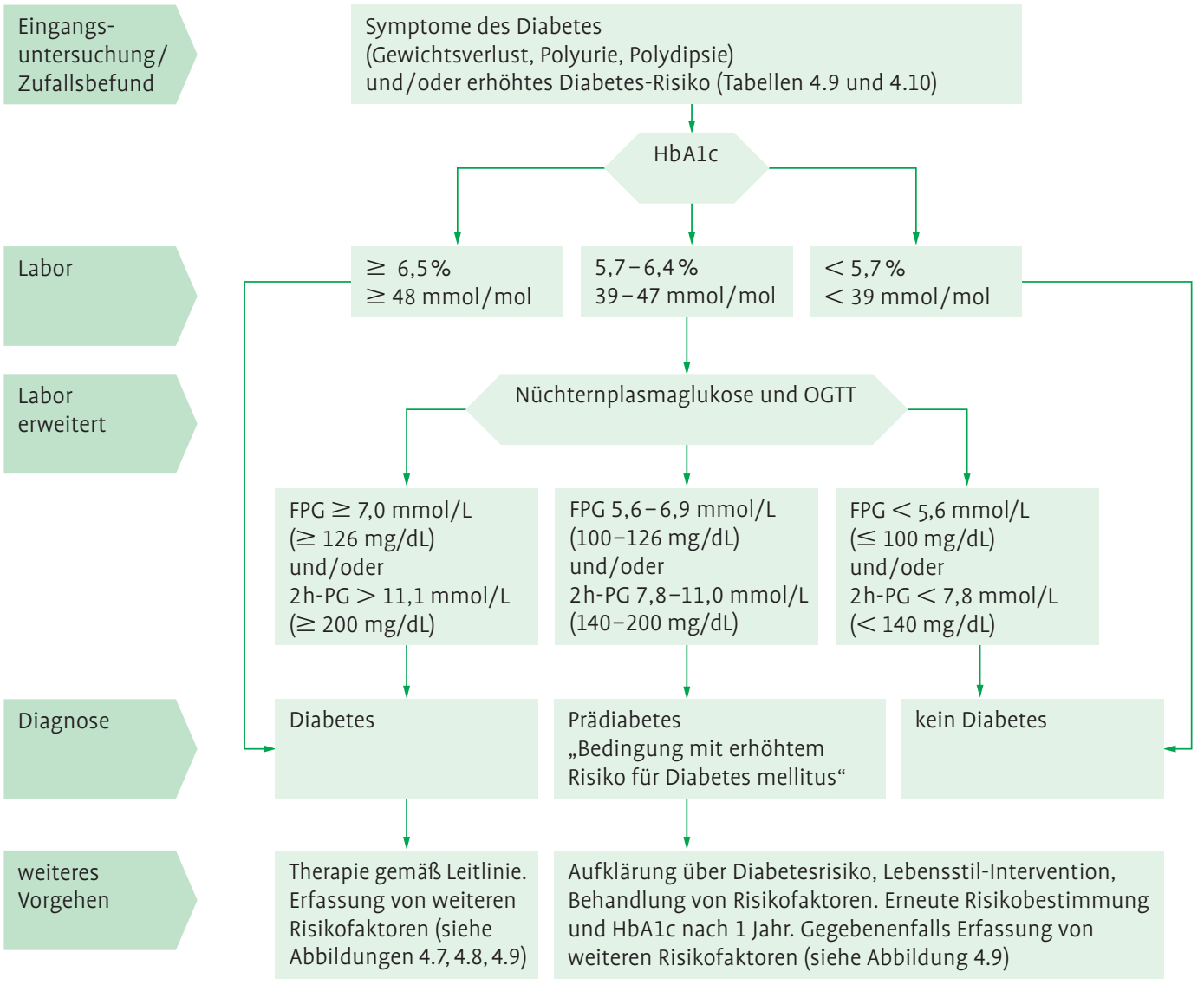

Abb. 4.5 Flussdiagramm zur Diagnose eines Diabetes mellitus (modifiziert nach Kerner et al. 2010 und http://www.deutsche-diabetes-gesellschaft.de/

redaktion/news/Stellungnahme_HbA1c_final.pdf); FPG = Nüchternplasmaglukose, OGTT = oraler Glukosetoleranztest, $2 \mathrm{~h}-\mathrm{PG}=2$-Stunden-Plasmaglukose nach OGTT. 
5. Bei Vorliegen von Diabetes-Symptomen (Gewichtsverlust, Polyurie, Polydipsie) soll der Diabetes primär durch Glukosemessungen diagnostiziert werden

6. Zustände, welche unabhängig von der Glykämie die HbA1c-Konzentration beeinflussen, erfordern ebenfalls die primäre Glukose-Messung:

a) Globinvarianten ( $\mathrm{HbS}, \mathrm{HbE}, \mathrm{HbF}, \mathrm{HbD}$, etc.)

b) Veränderte Lebensdauer von Erythrozyten (hämolytische Anämie, Eisenmangelanämie, Nierenerkrankungen, Lebererkrankungen, verstärkte Erythropoese im Rahmen der Anämie-Behandlung)

c) Chemische Modifikationen des Hämoglobins (carbamyliertes $\mathrm{Hb}$ bei Urämie, acetyliertes $\mathrm{Hb}$ bei hochdosierter und langfristiger Behandlung mit Vitamin C). d) Hemmung der Glykierung bei Dauertherapie mit Vitamin $\mathrm{C}$ oder $\mathrm{E}$.

e) Schwangerschaft (allerdings im Widerspruch zu den internationalen Emfehlungen) (zur Differenzierung von Gestationsdiabetes und Diabetes in der Schwangerschaft [s. Tab. 4.13]).

Die ADA empfiehlt, alle Personen jenseits des 45. Lebensjahres sowie unabhängig vom Allter alle Personen mit einem BMI $>25 \mathrm{~kg} / \mathrm{m}^{2}$, die einen oder mehrere der in $>$ Tab. 4.10 genannten Risikofaktoren aufweisen, auf das Vorliegen eines Diabetes mellitus zu untersuchen. Die DDG empfiehlt zudem die Untersuchung von Personen, welche beim Deutschen Diabetes Risikotest mehr als 40 Punkte haben (Tab. 4.9) (Schulze et al. 2007) (http://www.dife.de/ de/ presse/Diabetes_Test_Fragebogen.pdf).

Tab. 4.9 Erkennung von Personen mit erhöhtem Diabetes Risiko (http://www.dife.de/de/presse/Diabetes_Test_Fragebogen.pdf).

\begin{tabular}{lll}
\hline Parameter: Frage & Auswahlkriterien & Punkte \\
\hline Alter: & $<35$ & 0 Punkte \\
Wie alt sind Sie in Jahren? & $35-39$ & 1 Punkt \\
& $40-44$ & 3 Punkte \\
& $45-49$ & 5 Punkte \\
& $50-54$ & 7 Punkte \\
\hline Körperliche Aktivitäten: & Nein & 1 Punkt \\
Sind Sie pro Woche mindestens 5 Stunden aktiv? & Ja & 0 Punkte \\
(z. B. Sport, Gartenarbeit, Rad fahren) & Nein & 0 Punkte \\
\hline Bluthochdruck: & Ja & 5 Punkte \\
Wurde bei Ihnen schon einmal ein Bluthochdruck festgestellt? & 0 & 5 Punkte \\
\hline Vollkornbrotverzehr: & 1 & 4 Punkte \\
Wie viele Scheiben Vollkornbrot essen Sie am Tag? & 2 & 3 Punkte \\
& 3 & 2 Punkte \\
& 4 & 1 Punkt \\
& $>4$ & 0 Punkte \\
\hline Fleischkonsum: & Nie oder sehr selten & 0 Punkte \\
Wie oft essen Sie Rind-, Schweine- oder Lammfleisch & $1-2$ mal je Woche & 1 Punkt \\
(keine Wurstwaren)? & $3-4$ mal je Woche & 2 Punkte \\
Wie viele Tassen Kaffee trinken Sie am Tag? & $5-6$ mal je Woche & 4 Punkte \\
& Täglich & 5 Punkte \\
& Mehrmals täglich & 8 Punkte \\
\hline & $0-1$ & 2 Punkte \\
& $2-5$ & 1 Punkt \\
& $>5$ & 0 Punkte \\
\hline
\end{tabular}


Tab. 4.9 (Fortsetzung)

\begin{tabular}{|c|c|c|}
\hline Parameter: Frage & Auswahlkriterien & Punkte \\
\hline \multirow{5}{*}{$\begin{array}{l}\text { Rauchen: } \\
\text { Welchen Raucherstatus haben Sie? }\end{array}$} & Ich habe nie geraucht & O Punkte \\
\hline & $\begin{array}{l}\text { Ich habe mal durchschnittlich weni- } \\
\text { ger als } 20 \text { Zigaretten am Tag geraucht }\end{array}$ & 0 Punkte \\
\hline & $\begin{array}{l}\text { Ich habe mal durchschnittlich } 20 \mathrm{Zi} \text { - } \\
\text { garetten oder mehr am Tag geraucht }\end{array}$ & 3 Punkte \\
\hline & $\begin{array}{l}\text { Ich rauche durchschnittlich weniger } \\
\text { als } 20 \text { Zigaretten am Tag }\end{array}$ & 0 Punkte \\
\hline & $\begin{array}{l}\text { Ich rauche durchschnittlich } 20 \text { Ziga- } \\
\text { retten mehr am Tag }\end{array}$ & 6 Punkte \\
\hline
\end{tabular}

Alkohol: Ich trinke keinen oder nur gelegent- 2 Punkte Wie viele Gläser alkoholischer Getränke trinken Sie am Tag? lich Alkohol

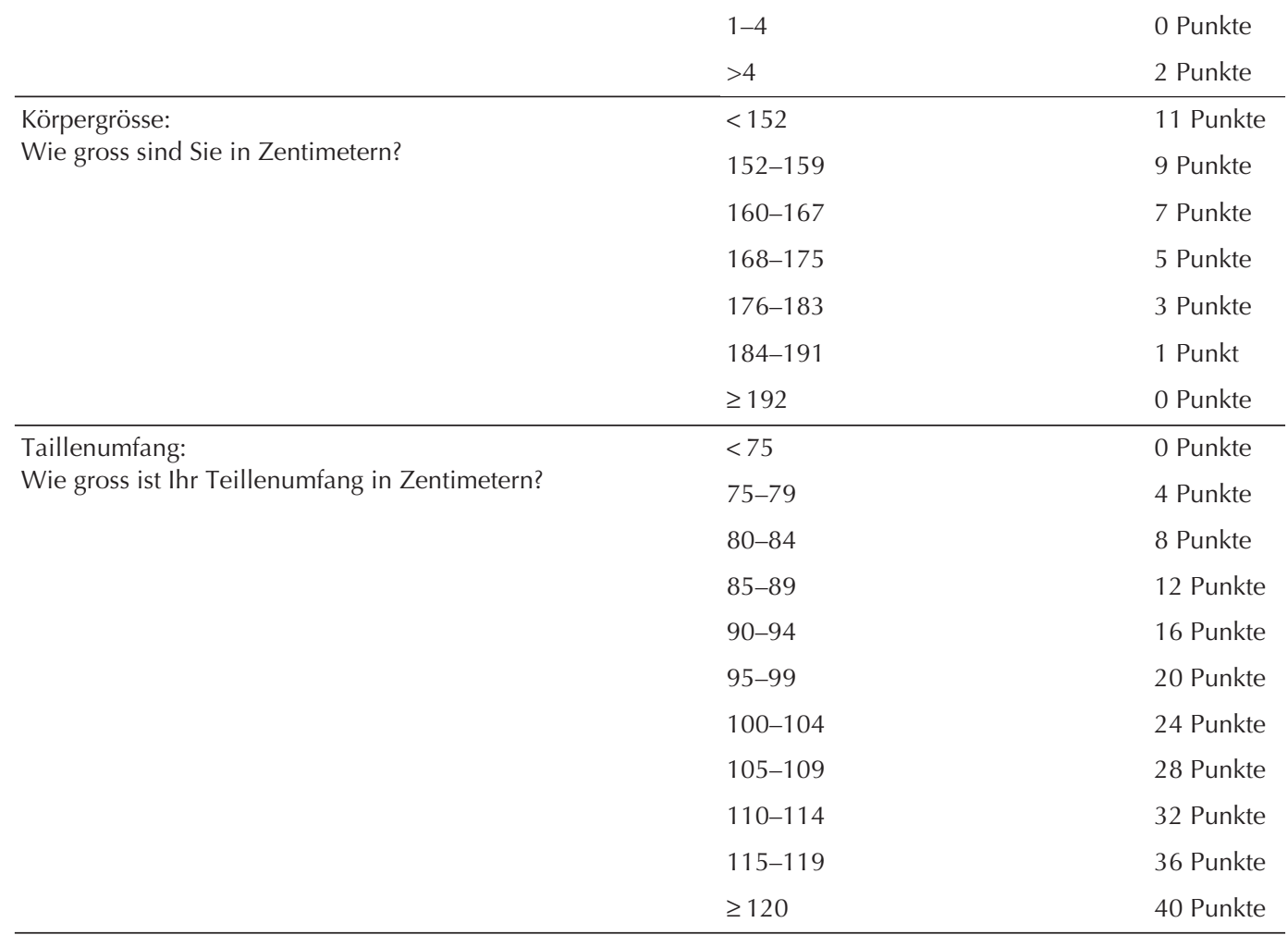

\section{Auswertung:}

0-29 Punkte: $\quad$ niedriges Diabetes-Risiko ( $<1 \%$ in 5 Jahren)

30-39 Punkte: $\quad$ noch niedriges Diabetes-Risiko (1-2 \% in 5 Jahren)

40-49 Punkte: $\quad$ erhöhtes Diabetes-Risiko (2-5\% in 5 Jahren)

50-59 Punkte: $\quad$ hohes Diabetes-Risiko (5-10\% in 5 Jahren).

>59 Punkte:

sehr hohes Diabetes-Risiko (> 10\% in 5 Jahren) 
Tab. 4.10 Personengruppen, bei denen die Suche nach einem Diabetes mellitus nach Empfehlungen der American Diabetes Association empfohlen ist (2010).

1. Erwachsene mit Übergewicht oder Adipositas (BMI $\left.\geq 25 \mathrm{~kg} / \mathrm{m}^{2 *}\right)$, die einen oder mehrere der folgenden Risikofaktoren aufweisen:

- körperliche Inaktivität

- Verwandte 1. Grades mit Diabetes

- Angehörige von Hochrisiko-Ethnien: (z. B., Afrikaner, Amerindianer, Lateinamerikaner, Asiaten, Ozeanier)

- Frauen, die ein Kind mit Geburtsgewicht > 4,5 kg geboren haben oder einen Schwangerschaftsdiabetes entwickelten

- Bluthochdruck ( $\geq 140 / 90$ mmHg oder anti-hypertensive Therapie)

- HDL Cholesterin <0,90 mmol/L (<35 mg/dL)

- Triglyzeride $>2,8 \mathrm{mmol} / \mathrm{l}(>250 \mathrm{mg} / \mathrm{dL})$

- Frauen mit Polyzystischem Ovarialsyndrom

- $\mathrm{HbA} 1 \mathrm{c} \geq 5,7 \%$ (> $39 \mathrm{mmol} / \mathrm{mol}$ ), IGT, oder IFG bei früherer Untersuchung

- andere klinische Bedingungen, die mit Insulinresistenz assoziiert sind (z. B. ausgeprägte Adipositas, Acanthosis nigricans)

- Anamnese mit kardiovaskulären Erkrankungen

2. Bei Fehlen der o. g. Kriterien sollte die Diabetes-Testung ab 45 Jahren beginnen

3. Bei normalem Testergebnis sollte die Untersuchung alle 3 Jahre wiederholt werden. Ggf. häufigere Untersuchungen in Abhängigkeit von Risikostatus (siehe 1.) oder Testergebnissen (siehe Tabelle 4.8).

Tab. 4.11 Differenzialdiagnostische Kriterien für Typ 1 und Typ 2 Diabetes mellitus (Kerner et al. 2010).

\begin{tabular}{|c|c|c|}
\hline & Typ 1 Diabetes mellitus & Typ 2 Diabetes mellitus \\
\hline Manifestationsalter & $\begin{array}{l}\text { meist Kinder, Jugendliche und junge } \\
\text { Erwachsene }\end{array}$ & $\begin{array}{l}\text { meist im mittleren und höheren } \\
\text { Erwachsenenalter }\end{array}$ \\
\hline Auftreten, Beginn & subakut bis akut & meist schleichend \\
\hline Symptome & $\begin{array}{l}\text { häufig Polyurie, Polydipsie, } \\
\text { Gewichtsverlust, Müdigkeit }\end{array}$ & häufig keine Beschwerden \\
\hline Körpergewicht & meist normgewichtig & $\begin{array}{l}\text { meist übergewichtig (abdominale } \\
\text { Adipositas) }\end{array}$ \\
\hline Ketoseneigung & ausgeprägt & fehlend oder gering \\
\hline Insulinsekretion & vermindert oder fehlend & initial subnormal bis hoch \\
\hline Insulinresistenz & keine & oft ausgeprägt \\
\hline familiäre Häufung & $\begin{array}{l}\text { gering (Konkordanz bei eineiigen } \\
\text { Zwillingen }<50 \% \text { ) }\end{array}$ & $\begin{array}{l}\text { typisch (Konkordanz bei eineiigen } \\
\text { Zwillingen }>50 \% \text { ) }\end{array}$ \\
\hline $\begin{array}{l}\text { Diabetes assoziierte Antikörper } \\
\text { (ICA, GAD65, IA-2, IAA)* }\end{array}$ & ca. $90-95 \%$ bei Manifestation & fehlend \\
\hline Lipoproteinstoffwechsel & unauffällig & $\begin{array}{l}\text { häufig } \\
\text { niedriges HDL-Cholesterin und er- } \\
\text { höhte Triglyzeride }\end{array}$ \\
\hline $\begin{array}{l}\text { Ansprechen auf betazytotrope Anti- } \\
\text { diabetika }\end{array}$ & meist fehlend & zunächst meist gut \\
\hline Insulintherapie & erforderlich & $\begin{array}{l}\text { meist erst nach jahrelangem Verlauf } \\
\text { der Erkrankung wegen nachlassender } \\
\text { Insulinsekretion erforderlich }\end{array}$ \\
\hline
\end{tabular}

ICA = Inselzellantikörper, GAD65 = Antikörper gegen Glutamat-Decarboxylase, IA-2 = Antikörper gegen Tyrosin-Phosphatase 2, IAA = Insulin-Autoantikörper 
Im Erwachsenenalter manifestiert sich zumeist ein Typ 2 Diabetes mellitus, aber es kommen auch Spätmanifestationen eines Typ 1 Diabetes mellitus vor $(\mathrm{LADA}=$ latent insulinpflichtiger Diabetes im Erwachsenenalter). $>$ Tab. 4.11 stellt die differentialdiagnostischen Kriterien dieser beiden hauptsächlichen Diagnosen gegenüber. Bei Verdacht auf Typ 1 Diabetes mellitus empfiehlt sich die Untersuchung von anti-Glutamatdecarboxylase Antikörpern (anti-GAD65), welche bei 90-95\% der Typ 1 Diabetiker vorkommen (Kerner et al. 2010).

\subsubsection{Schwangerschaftsdiabetes (GDM)}

Die frühe Diagnose eines Schwangerschaftsdiabetes (Gestationsdiabetes mellitus = GDM) und die damit verbundene richtige und optimale Behandlung verbessert die Prognose von Mutter und Kind. Die optimale Kontrolle des Diabetes in der Schwangerschaft bedeutet die Erreichung der Euglykämie in allen Stadien der Schwangerschaft, wie auch vor der Konzeption (bei vorbestehendem Diabetes) und nach der Geburt. Für dieses Ziel wurden von der International Association of Diabetes and Pregnancy Study Groups (IADPSG), welche sich aus Geburtshelfern, Pädiatern, Diabetologen und Epidemiologen zusammensetzt, neue internationale Guidelines veröffentlicht (International Association of Diabetes and Pregnancy Study Groups Consensus Panel 2010; Costorino et al. 2011). Die Tabellen 4.12 und 4.13 fassen die Schwellenwerte für die Diagnose eines GDM bzw. manifesten Diabetes mellitus in der Schwangerschaft sowie die Strategien für deren Erkennung ( Abb. 4.6) zusammen (International Association

Tab. 4.12 Schwellenwerte für die Diagnose eines Schwangerschaftsdiabetes (International Association of Diaetes and Pregnancy Study Groups 2010: Castorino et al. 2011).

\begin{tabular}{lll}
\hline Messgrösse & \multicolumn{2}{l}{$\begin{array}{l}\text { Schwellenwert für } \\
\text { Glucosekonzentration* }\end{array}$} \\
\hline & $\mathrm{mmol} / \mathrm{L}$ & $\mathrm{mg} / \mathrm{dL}$ \\
Nüchtern-Plasma-Glukose & 5.1 & 92 \\
$\begin{array}{l}\text { 1-h Plasma Glucose (75g } \\
\text { OGTT) }\end{array}$ & 10.0 & 180 \\
2-h Plasma-Glucose & 8.5 & 153 \\
\hline * Einer oder mehrere dieser Schwellenwerte müssen für \\
$\begin{array}{l}\text { die Diagnose eines Schwangerschaftsdiabetes überschrit- } \\
\text { ten werden. }\end{array}$
\end{tabular}

Tab. 4.13 Schwellenwerte für die Diagnose eines Diabetes mellitus in der Schwangerschaft (International Association of Diaetes and Pregnancy Study Groups 2010: Castorino et al. 2011).

\begin{tabular}{ll}
\hline Messgrösse & Konsensus-Schwellenwert \\
\hline FPG $^{1}$ & $\geq 7,0 \mathrm{mmol} / \mathrm{L}(>126 \mathrm{mg} / \mathrm{dL})$ \\
$\mathrm{HbA} \mathrm{c}^{2}$ & $\geq 6,5 \%$ nach NGSP, $>48$ \\
& $\mathrm{mmol} / \mathrm{mol}$ nach IFCC \\
$\begin{array}{l}\text { zufällig bestimmte } \\
\text { Plasma-Glucose- } \\
\text { Konzentration }\end{array}$ & $+11,1 \mathrm{mmol} / \mathrm{L}(200 \mathrm{mg} / \mathrm{dL})$ \\
\end{tabular}

1 Eines der beiden Kriterien muss erfüllt sein, um einen manifesten Diabetes in der Schwangerschaft zu diagnostizieren.

2 Wenn durch eine zufällige (postprandiale) Untersuchung eine Glukose-Plasmakonzentration oberhalb dieses Schwellenwertes bestimmt wird, muss die Verdachtsdiagnose durch FPG oder HbA1c bestätigt werden.

of Diabetes and Pregnancy Study Groups Consensus Panel 2010; http://www.deutsche-diabetes-gesellschaft.de/ redaktion/news/EbLL_GDM_ENDFASSUNG_2011_01_28_E1.pdf).

Im Unterschied zu früheren Empfehlungen, wird aufgrund der Ergebnisse der Hyperglycemia and Adverse Pregnancy Outcome (HAPO) Studie ein Diabetes-Screening für alle Schwangeren und die direkte Anwendung des 75g-OGTT in der 24.28. Schwangerschaftswoche empfohlen. Der früher eingesetzte 50g-OGTT wird als obsolet definiert, vor allem weil das dadurch induzierte 2-Schritt-Vorgehen die Diagnose und damit auch Behandlung des GDM in die 30.-34. Schwangerschaftswoche verzögert, wo die Effekte von von Hyperglykämie und Makrosomie bereits wirken. Neu ist auch die Differenzierung von GDM und manifestem Diabetes. Es ist wahrscheinlich, dass diese Empfehlungen von der ADA in 2011 übernommen werden. Andere nationale Übernahmen in originaler oder adaptierter Version werden folgen (http://www.deutsche-diabetes-gesellschaft.de/ redaktion/news/EbLL_GDM_ENDFASSUNG_2011_ 01_28_E1.pdf).

In Europa und Deutschland wird sicherlich ein wichtiger Aspekt sein, ob alle Frauen oder nur Frauen aus zu definierenden Risikogruppen eingeschlossen werden. Eine großzügige Anwendung erscheint notwendig, um Schwangerschaftskomplikationen (z. B. Malformationen, Fehlgeburten), aber auch das erhöhte Risiko von makrosomalen Kindern für Adipositas, Diabetes und Herz-Kreis- 


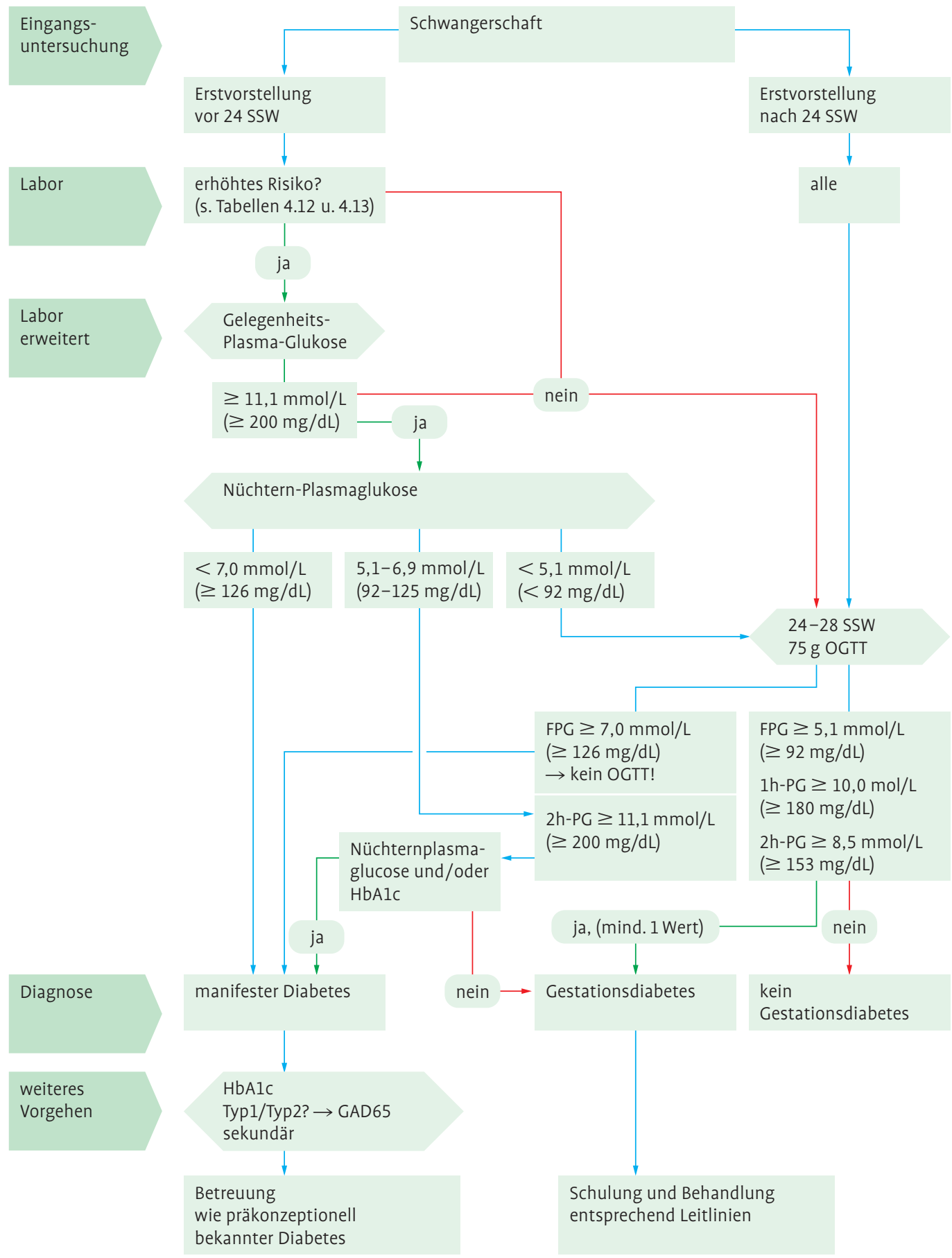

Abb.4.6 Flussdiagramm zur Diagnose eines Schwangerschaftsdiabetes oder Diabetes mellitus in der Schwangerschaft (modifiziert nach http://www.deutsche-diabetes-gesellschaft.de/redaktion/news/

EbLL_GDM_ENDFASSUNG_2011_01_28_E1.pdf);
1: Risikopersonen sind in Tabellen 3 und 4 beschrieben; SSW = Schwangerschaftswoche, FPG = Nüchternplasmaglukose, OGTT = oraler Glukosetoleranztest, 2h-PG = 2-Stunden-Plasmaglukose nach OGTT, GAD65 = Antikörper gegen Glutamat-Decarboxylase 65 . 
lauferkrankungen im Kindes- und/oder Erwachsenenalter zu minimieren

Frauen mit GDM haben ein erhöhtes Risiko von ca. $30 \%$ innerhalb von 5 Jahren postpartum einen manifesten Diabetes zu entwickeln (Ekelund et al. 2010). HbA1c und FPG während der Schwangerschaft sowie die Zahl früherer Schwangerschaften und eine Familiengeschichte für Diabetes mellitus sind unabhängige Risikofaktoren für das spätere Eintreten eines postpartum Diabetes mellitus. Ein $\mathrm{HbA} 1 \mathrm{c}>5,7 \%$ (> $39 \mathrm{mmol} / \mathrm{mol})$ und eine FPG $>5,2 \mathrm{mmol} / \mathrm{l}$ erhöhen das Diabetes-Risiko um die Faktoren 4 bzw. 6. Ensprechend müssen diese Frauen in Bezug auf ihr erhöhtes Diabetes Risiko aufgeklärt und bezüglich ihrer Glykämie regelmässig untersucht werden. 10\% der Frauen mit GDM haben Inselzellantikörper und ein erhöhtes Risiko für die Entwicklung eines Typ 1 Diabetes mellitus.

\subsubsection{Monitoring des Diabetes Mellitus Glykämie-Kontrolle}

Kardiovaskuläre Komplikationen sind die Hauptursache für Morbidität und Mortalität von Patienten mit Diabetes mellitus. Der Diabetes Control and Complications Trial (DCCT) und die United Kingdom Prospective Diabetes Study (UKPDS) wiesen übereinstimmend nach, dass die intensive glykämische Kontrolle die Entwicklung und Progression von mikrovaskulären Komplikationen von Patienten mit Typ 1 oder Typ 2 Diabetes günstig beinflusst. Umstritten ist jedoch, ob intensive Glukose-Senkung auch makrovaskuläre Ereignisse verhütet. Bei längerer Nachbeobachtung der beiden o.g. Studien stellte sich heraus, dass intensive glykämische Kontrolle die Inzidenz von Herzinfarkt und Tod aufgrund kardiovaskulärer Ursachen senkt. Im Gegensatz dazu fanden die ACCORD-, ADVANCE- und VADT-Studien, dass intensive glykämische Kontrolle entweder keinen oder sogar einen schädlichen Effekt auf kardiovaskuläre Ereignisse hat (Brown et al. 2010). Auch die Ergebnisse einer britischen Registerstudie bei nahezu 48'000 Diabetikern sprechen dafür, dass eine zu scharfe glykämische Kontrolle die Gesamtsterblichkeit und die Rate kardiovaskulärer Ereignisse erhöht (Curie et al. 2010). Die adjustierten HbA1c-Werte zeigten eine $U$-förmige Beziehung mit der Gesamtsterblichkeit. Die niedrigste Sterblichkeit fand sich mit der Dezile assoziert, welche das $\mathrm{HbA} 1 \mathrm{c}-$ Intervall 7,5-7,6 \% (55 $\mathrm{mmol} / \mathrm{mol}-56 \mathrm{mmol} / \mathrm{mol}$ ) umfasst. Trotz dieser alarmierenden Studienergebnisse hält die DDG derzeit noch an dem Behandlungsziel für $\mathrm{HbA} 1 \mathrm{c}$ $<6,5 \%(<48 \mathrm{mmol} / \mathrm{mol}$ ) fest $(>$ Abb. 4.7) (Matthaei et al. 2010), statt $<7 \%$ (52 $\mathrm{mmol} / \mathrm{mol})$ in anderen Ländern. Sie schränkt allerdings ein, dass der Vermeidung von Nebenwirkungen (Hypoglykämien, wesentliche Gewichtszunahme) eine hohe Priorität zukommt, so dass der HbA1c-Zielwert bei 7,0 (52 $\mathrm{mmol} / \mathrm{mol}$ ) belassen werden sollte, wenn ein Zielwert von $<6,5 \%(<48 \mathrm{mmol} / \mathrm{mol})$ nur mit den o. g. Nebenwirkungen erreicht werden kann.

\section{Mikroalbuminurie und eGFR}

Diabetes mellitus ist die häufigste Ursache einer chronischen Niereninsuffizienz. 20-40 \% der Diabetiker entwickeln eine chronische Nierenerkrankung. Auch wenn der Begriff „Diabetische Nephropathie" in Bezug auf Ätiologie und Pathogenese unterschiedliche Krankheitsbilder zusammenfasst, so münden diese in der Regel unbehandelt in eine terminale Niereninsuffizienz, welche eine Nierenersatztherapie notwendig macht. Frühformen der diabetischen Nephropathie äußern sich in einer selektiven glomerulären Proteinurie, welche als Mikroalbuminurie bezeichnet wird. Je nach Untersuchungsmethode (Albumin +/- Kreatinin) und -material (Spontan- oder Sammelurin) und allenfalls Sammelperiode (befristet oder 24 Stunden) sind unterschiedliche diagnostische Cut-Offs definiert $(\checkmark$ Tab. 4.14). Die Mikroalbuminurie ist nicht nur ein Risikofaktor für die Progression der diabetischen Nephropathie sondern zeigt auch ein um Faktor 2 erhöhtes Risiko an, einer kardiovaskulären Krankheit zu erkranken oder zu versterben. Das Ausmaß der Albuminurie und die Progression der diabetischen Nephropathie sind durch mehrere Risikofaktoren moduliert ( $\triangleright$ Tab. 4.15), die zum Teil therapeutisch beeinflusst werden können, vor allem durch die Optimierung der Glykämie, des Blutdrucks, des Körpergewichtes, der Plasmakonzentrationen von LDL-Cholesterin, HDL-Cholesterin und Triglyzeriden sowie durch Nichtrauchen. Die internationalen und nationalen Richtlinien empfehlen die einmal jährliche Untersuchung von Diabetikern auf Mikroalbuminurie ( Abb.4.8) (http://www.versorgungsleitlinien.de/ themen/diabetes2/dm 2_nephro/pdf/nvl_t2dnephro_lang.pdf).

Bei Vorliegen einer Mikroalbuminurie müssen die ohnehin geltenden Therapieziele intensiviert verfolgt werden: $\mathrm{HbA} 1 \mathrm{c}<6,5 \% \mathrm{bzw} .<48 \mathrm{mmol} / \mathrm{mol}$ (ggf. $<7 \%$ bzw. $<52 \mathrm{mmol} / \mathrm{mol}$ ), Blutdruck $<135$ 140/80 mm Hg und LDL-Cholesterol < 1,8 mmol/L 


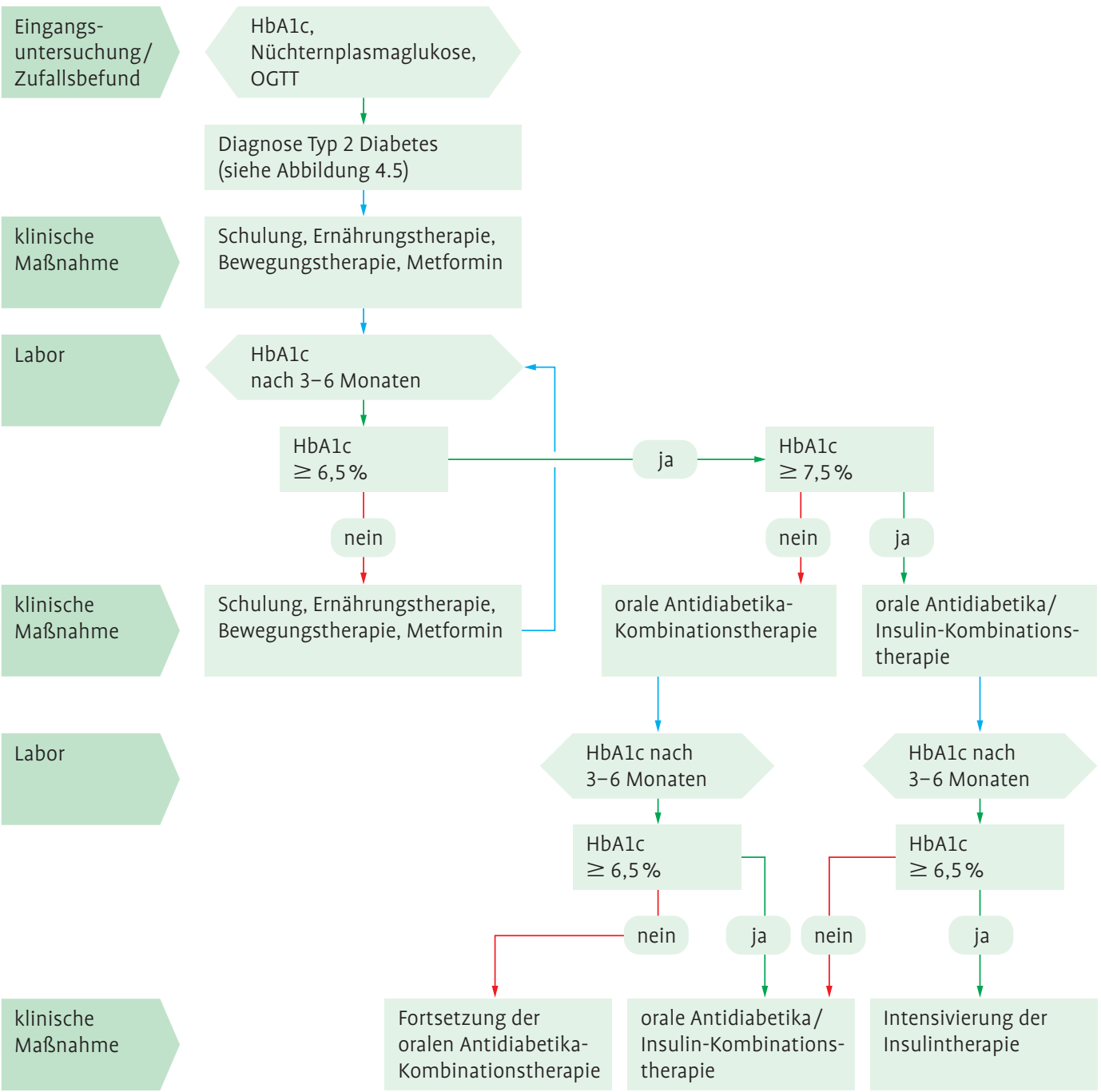

Abb.4.7 Flussdiagramm zum Monitoring der Glykämie bei Diabetes mellitus (modifiziert nach Mattahaei et al. 2010: http://www.deutsche-diabetes-gesellschaft.de/

$(<70 \mathrm{mg} / \mathrm{dL}$ ). Aufgrund der Ergebnisse kontrollierter Studien wird der Einsatz von Angiotensin Converting Enzyme Inhibitoren oder Angiotensin-II-Rezeptorblockern für die antihypertensive Therapie von Diabetikern mit Mikroalbuminurie empfohlen.

\section{Lipidstoffwechsel}

Patienten mit Diabetes mellitus Typ 2 (nicht Typ 1) unterscheiden sich von nicht-diabetischen Perso-
redaktion/mitteilungen/leitlinien/PL_DDG2010_ Behandlung_Typ2.pdf).

nen gleichen Alters und Geschlechts durch eine höhere mittlere Plasmakonzentration von Triglyzeriden und eine niedrigere mittlere HDL-Cholesterin-Konzentration. Häufig findet sich das NiedrigHDL-Cholesterin/Hypertriglyzeridämie-Syndrom schon vor der Manifestation eines Diabetes mellitus, weswegen Patienten mit dieser Dyslipidämie eingehend auf das Vorliegen eines Diabetes mellitus untersucht werden sollten, inkl. Durchführung eines OGTT $(\triangleright$ Tab. 4.10). 
Tab. 4.14 Diagnostische Cut-Offs* für die Albuminurie in Abhängigkeit vom Probenmaterial.

\begin{tabular}{|c|c|c|c|c|c|}
\hline & \multicolumn{2}{|c|}{ Sammelurin } & \multicolumn{3}{|c|}{ Spontanurin } \\
\hline & $(\mathrm{mg} / 24 \mathrm{~h})$ & $\mu \mathrm{g} / \mathrm{min}$ & $\mathrm{mg} / \mathrm{l}$ & $\mathrm{mg} / \mathrm{mmol}$ Kreatinin & mg/g Kreatinin \\
\hline normal & $<30$ & $<20$ & $<20$ & $<2$ & $<30$ \\
\hline Mikroalbuminurie & $30-300$ & 20-200 & $20-200$ & $2-20$ & $30-300$ \\
\hline Makroalbuminurie & $>300$ & $>200$ & $>200$ & $>20$ & $>300$ \\
\hline
\end{tabular}

* Für Frauen werden teilweise um 1/3 tiefere Cut-Offs verwandt

Tab. 4.15 Risikofaktoren der diabetischen Nephropathie.

\begin{tabular}{ll}
\hline Beeinflussbare Risikofaktoren & Nicht beeinflussbare Risikofaktoren \\
\hline Hyperglykämie & höheres Alter \\
Bluthochdruck & männliches Geschlecht \\
Albuminausscheidungsrate & Dauer der Diabeteserkrankung \\
Rauchen & Beginn des Diabetes vor dem 20. Lebensjahr \\
erhöhtes LDL-Cholesterin & gleichzeitiges Vorliegen einer Retinopathie \\
Hypertriglyzeridämie & positive Familienanamnese für Hypertonie und/oder Nephropatie \\
erniedrigtes HDL-Cholesterin & ethnische Herkunft: Afrikaner, Lateinamerikaner, indigene \\
erhöhter body mass index & Amerikaner \\
\hline
\end{tabular}

Die Plasmakonzentrationen von LDL- und HDLCholesterin wie auch der Triglyzeride beeinflussen die Risiken von Diabetikern für sowohl makro- als auch mirkrovaskuläre Erkrankungen. Entsprechend ist die Korrektur dieser Lipidstoffwechselstörungen eine wichtige Massnahme für die Reduktion der kardiovaskulären Risiken von Diabetikern. Tatsächlich haben mehrere Studien gezeigt, dass die Absenkung des LDL-Cholesterin das koronare Risiko von Diabetikern senkt (Cholesterol Treatment Trialists Collaborators et al. 2008), so dass für Diabetiker genauso tiefe Zielwerte für LDL-Cholesterin wie für Patienten mit manifester Atherosklerose definiert sind, nämlich $<2,6 \mathrm{mmol} / \mathrm{L}(<100 \mathrm{mg} / \mathrm{dL})$ oder gar $<1,8 \mathrm{mmol} / \mathrm{L}(<70 \mathrm{mg} / \mathrm{dL}$ ) (www.chd-taskforce.de/guide.htm; The Task Force for the management of dyslipidaemias 2011).

Der klinischen Nutzen der Behandlung von Hypertriglyzeridämie und niedrigem HDL-Cholesterin stellt sich komplexer dar: Die Behandlung mit Fibraten, allein oder in Kombination mit Statinen, zeigte keinen Nutzen in Bezug auf die Absenkung des kardiovaskulären Risikos (Jun et al. 2010), wohl aber in Bezug auf die Absenkung der Risiken für Nephropathie und Retinopathie (Fioretto et al. 2010). Außerdem sprechen die Ergebnisse von posthoc Analysen dafür, dass Diabetiker mit HDL-Cho- lesterin $<1 \mathrm{mmol} / \mathrm{L}(<39 \mathrm{mg} / \mathrm{dL})$ und Triglyzeriden $>2.3 \mathrm{mmol} / \mathrm{L}$ (> $200 \mathrm{mg} / \mathrm{dL}$ ) unter Behandlung mit Fibraten seltener Herzinfarkte erleiden (24).

Als Konsequenz dieser Daten soll bei Diabetikern mindestens einmal pro Jahr der Lipidstatus erhoben werden ( $\triangleright$ Abb. 4.9).

\subsubsection{Lipid- und Lipoproteinstoffwechsel}

\subsubsection{Abschätzung des kardiovaskulären Risikos}

In vielen epidemiologischen Studien und einer Meta-Analyse der Originaldaten aus diesen Studien (Emerging Risk Factors Collaboration et a. 2009) war die Inzidenz von koronaren Ereignissen positiv mit den Plasmakonzentrationen von Gesamt-Cholesterin, LDL-Cholesterin, nonHDL-Cholesterin und Triglyzeriden sowie invers mit dem HDL-Cholesterin korreliert. Die Lipid-Risikofaktoren interagieren miteinander und mit anderen Risikofaktoren, so dass beim Vorliegen mehrerer Risikofaktoren das kardiovaskuläre Risiko überproportional ansteigt. Tatsächlich ist das kardiovaskuläre Risiko beim Vorliegen mehrerer mittelgradig ausgeprägter Risikofaktoren häufig höher als bei starker Ausprägung eines einzelnen. Für eine mög- 
Mikroalbuminurie und geschätzte Glomeruläre Filtrationsrate (eGFR) bei Diabetes mellitus
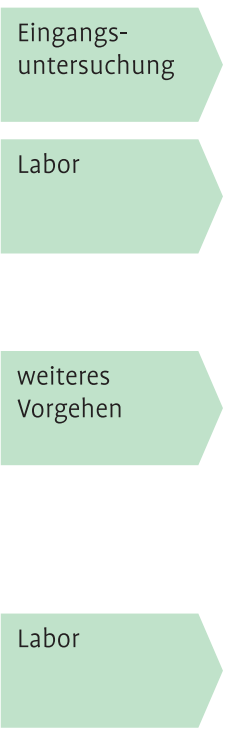

Diagnose Diabetes

Bestimmung der Albumin-Kreatinin-Ratio (AKR) im Urin und der eGFR (Kreatinin im Plasma)

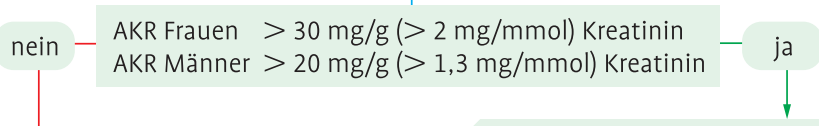

gibt es Faktoren, die die Albuminausscheidung beeinflusst haben (nichtrenale Ursachen)? Urinstreifentest

behandelbare Faktoren behandeln / bei sonstigen warten bis Rückgang zweite Bestimmung der AKR ( $<3$ Monaten)

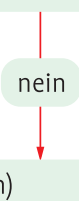

zWeite Bestimnung der AKR $(<3$ Monaten)

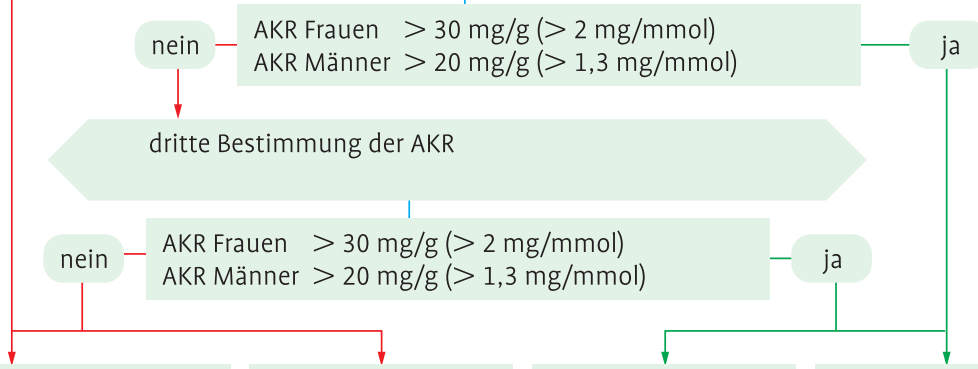

Albuminurie neg. und eGFR $\geq 60 \mathrm{~mL} / \mathrm{min}$

Albuminurie neg. und eGFR $<60 \mathrm{~mL} / \mathrm{min}$

Albuminurie pos. und eGFR $<60 \mathrm{~mL} / \mathrm{min}$

Albuminurie pos. und eGFR $\geq 60 \mathrm{~mL} / \mathrm{min}$

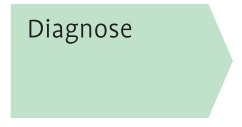

weiteres Vorgehen kein Hinweis für

diabetische

Nephropathie

Screening in einem Jahr
Weitere Abklärung: Urinsediment, Nierensonographie, gegebenenfalls nephrologisches Konsil

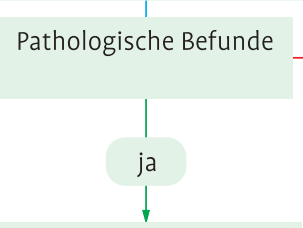

V.a. nichtdiabetische Nephropathie

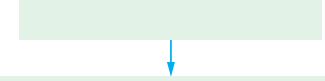

weitere diagnostische Abklärung durch Nephrologen

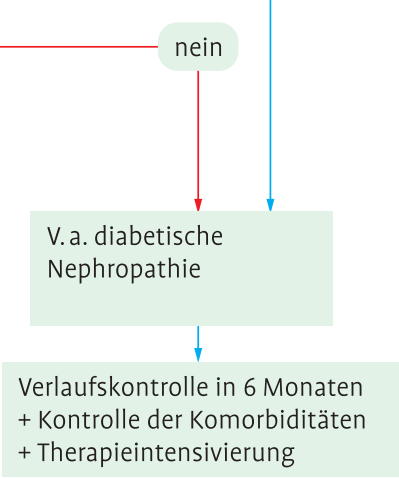

Abb.4.8 Flussdiagramm zur Diagnose der diabetischen Nephropathie (modifiziert nach http://www.versorgungsleitlinien.de/themen/diabetes2/dm 2_nephro/ pdf/nvl_t2dnephro_lang.pdf); AKR = Albumin/Kreatinin Ratio im Urin, eGFR = geschätzte glomeruläre Filtrationsrate. 
Erhebung eines Lipidstatus

Erstuntersu-
chung/Klinischer
Befund

Labor

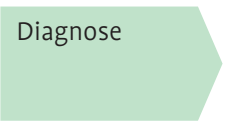

Labor erweitert

\section{weiteres}

Vorgehen
Eigen- und Familienanamnese körperliche Untersuchung

Symptomatische Arteriosklerose: KHK, CVK, PAVK?

bekannter Diabetes mellitus

bekannte Niereninsuffizienz

ja

nein

$\checkmark$

Alter $>45$ Jahre, BMI $>25 \mathrm{~kg} / \mathrm{m}^{2}$ oder Taillenumfang $>94 \mathrm{~cm}(\mathrm{~m}), 80 \mathrm{~cm}(\mathrm{f})$ positive Familienanamnese für Diabetes mellitus oder frühzeitige Arteriosklerose Hypertonie bekannt oder gemessen, Raucher

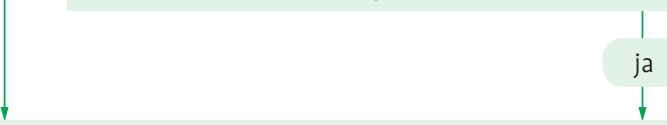

Lipidstatus: Gesamt-, LDL-, HDL-Cholesterin, Triglyzeride Glukose nüchtern oder HbAlc

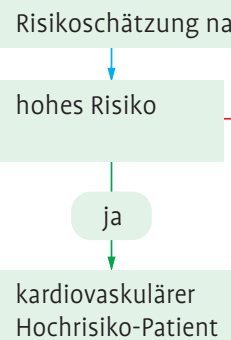

Abbildungen 4.10 und 4.11

Hochrisiko-Patient

weitere Risikofaktoren

- Lp (a) • (hs Troponin)

- hs CRP • ([NT-Pro] BNP) • (Albumin im Urin)

Lp (a) $>300 \mathrm{ng} / \mathrm{L} \quad$ hs CRP $>2 \mathrm{mg} / \mathrm{L}$

(hs Troponin $>99$. Perzentile)

(NT pro BNP > $300 \mathrm{ng} / \mathrm{L}),(\mathrm{BNP}>100 \mathrm{ng} / \mathrm{L})$

(Mikroalbuminurie)

ja

intermediäres oder niedriges Risiko

kardiovaskulärer Niedrigrisiko-Patient

unauffälliger Befund 
lichst zuverlässige Abschätzung des kardiovaskulären Risikos sollen also die klassischen Risikofaktoren zusammen erfasst werden und mit Hilfe von Scores, Tafeln oder im Internet zugänglichen Algorithmen berechnet werden. Für Deutschland werden zumeist die Empfehlungen der International Atherosclerosis Society (PROCAM-Score und -Algorithmus) (www.chd-taskforce.de/guide.htm; von Eckardstein et al. 2005) oder die Joint European Guidelines (The Task Force for the management of dyslipidaemias 2011) bzw. ihre für Deutschland modifizierte Form (D-SCORE) angewandt (Keil et al. 2005). ( Abb. 4.9 bis 4.11).

Bei Patienten mit mittlerem Risiko (10-20\% Risiko für Koronareignisse, $>1 \%$ kardiovaskuläres Mortalitätsrisiko wird die Untersuchung zusätzlicher Risikofaktoren, insbesondere sensitives CRP aber auch Lp(a) empfohlen (Assmann et al. 2005; Noordestgaard 2010), um allenfalls aggressivere Therapieziele zu rechtfertigen. Darüberhinaus haben sich in epidemiologischen Studien Biomarker für durch Arteriosklerose bedingte Organschäden (sensitive kardiale Troponine T oder I, B-Typ natriuretische Peptide (BNP oder NT-proBNP) sowie Mikroalbuminurie und Cystatin $C$ als besonders informativ für die Reklassifizierung des kardiovaskulären Risikos erwiesen (Blankenberg et al. 2010) ( Abb. 4.9). Die Absenkung der LDL-Cholesterin-Konzentration vor allem durch Statine senkt das Herzinfarktrisiko dosisabhängig. In einer jüngst publizierten MetaAnalyse der Daten von 170000 Teilnehmern an 26 randomisierten und kontrollierten Endpunktstudien von mindestens 2 jähriger Nachbeobachtungsdauer zeigte (Cholesterol Treatment Trialists Collaboration et al. 2010), dass mit jeder Absenkung des LDL-Cholesterins um $1 \mathrm{mmol} / \mathrm{L}(38,4 \mathrm{mg} / \mathrm{dl})$ die jährliche Rate von tödlichen und nichttödlichen Koronarereignissen um ca. 20\% reduziert wurde. Eine mit modernen Statinen erreichbare Absenkung des LDL-Cholesterins um 2-3 mmol/L (77-115 mg/dL) würde die Herzinfarkt-Inzidenz um $40-50 \%$ senken. In den bisherigen Studien wurde kein unterer Schwellenwert für LDL-Cholesterin gefunden, unterhalb dessen das Risiko plafonierte oder sogar wieder stieg (anders als bei Glukose- oder Blutdruck-Senkung). Auch wurde keine Erhöhung der Inzidenzen von anderen schweren Erkrankungen wie Krebs oder anderen Todesursachen gefunden, so dass auch die Gesamtsterblichkeit sank (Cholesterol Treatment Trialists Collaboration et al. 2010). Als Ergebnis dieser Studien wird heute für Patienten mit mani-

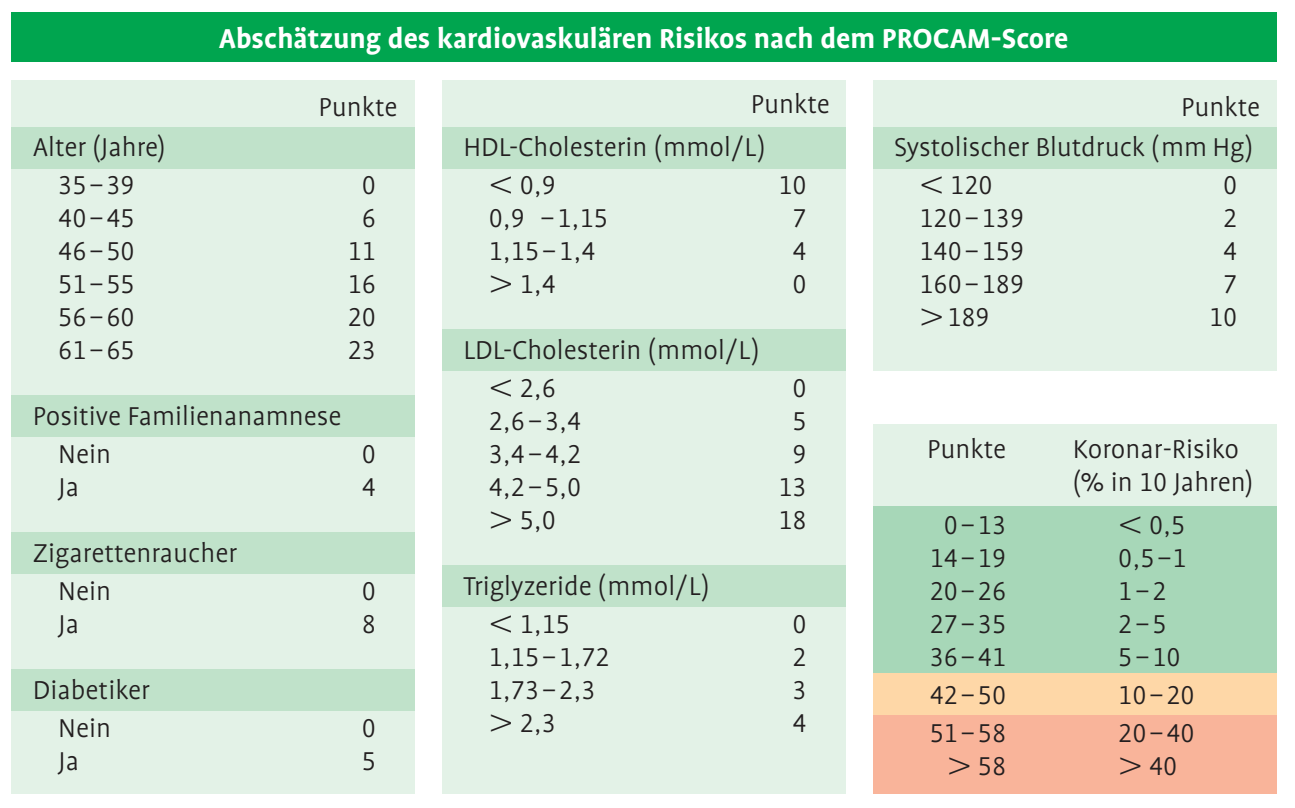

Abb.4.10 Abschätzung des kardiovaskulären Risikos nach Emfehlungen der Internationalen ArterioskleroseGesellschaft (von Eckardstein et al. 2005): präzisere Be- rechnung via http://www.assmann-stiftung.de/stiftungsinstitut/procam-tests/procam-gesundheitstest/. 


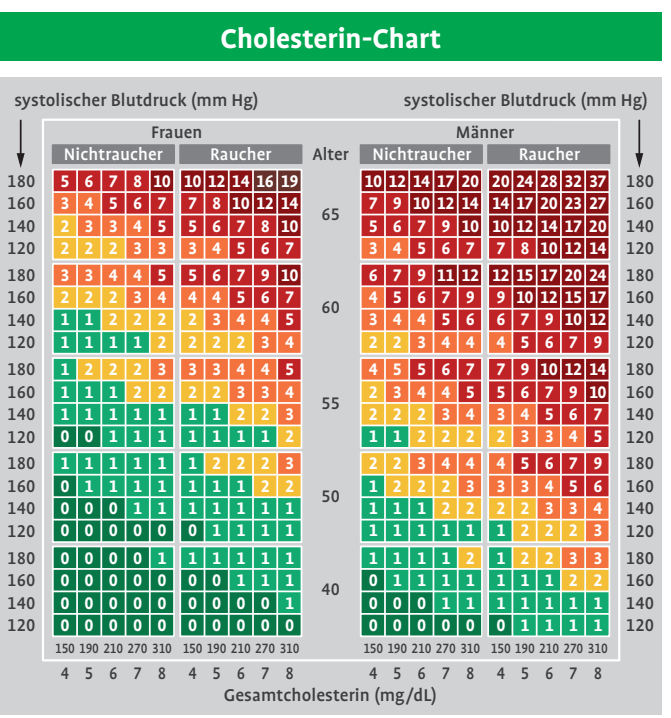

Abb. 4.11 Abschätzung des kardiovaskulären Risikos nach den Gemeinsamen Europäischen Empfeh-

\section{Cholesterin/HDL-Cholesterin-Ratio}

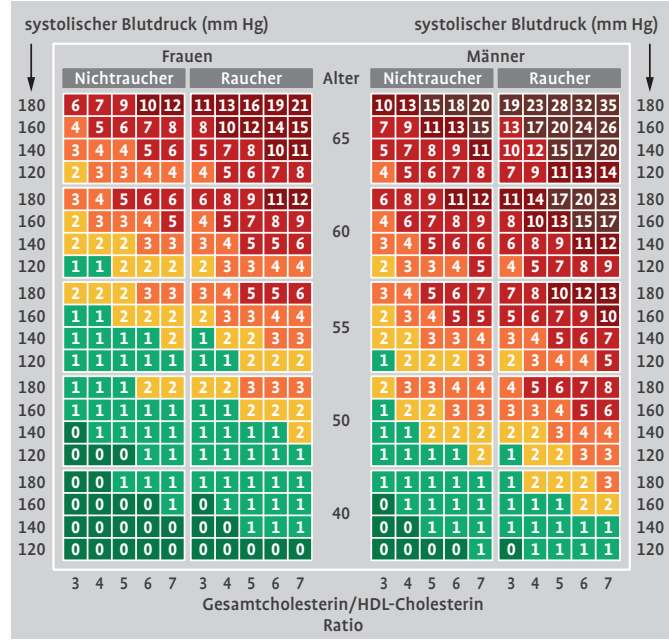

lungen (modifiziert für Deutschland, Keill et al. 2005).

Tab. 4.16 Kardiovaskuläre Risikostufen und Zielwerte für LDL-Cholesterin (The Task Force for the management of dyslipidaemias 2011, von Eckardstein et al. 2005).

\begin{tabular}{lllll}
\hline IAS-Empfehlung: & Joint European Guidelines: & Ein- & Zielwert für LDL- & Behandlung \\
Risiko für ein kardio- & Risiko für ein tödliches & stufung & Cholesterin &
\end{tabular}

vaskuläres Ereignis kardiovaskuläres Ereignis

(tödlich oder nicht- in den nächsten 10 Jahren

tödlich) in den

nächsten 10 Jahren

\begin{tabular}{|c|c|c|c|c|}
\hline$<10 \%$ & $<1 \%$ & niedrig & $\begin{array}{l}<4,1 \mathrm{mmol} / \mathrm{L} \\
(<160 \mathrm{mg} / \mathrm{dL})\end{array}$ & $\begin{array}{l}\text { In der Regel Motivation zu } \\
\text { Lebensstil- und Verhaltens- } \\
\text { änderungen }\end{array}$ \\
\hline $10-20 \%$ & $1-5 \%$ & mittel & $\begin{array}{l}<3,4 \mathrm{mmol} / \mathrm{L} \\
(<130 \mathrm{mg} / \mathrm{dL})\end{array}$ & $\begin{array}{l}\text { Wie oben plus mglw. Me- } \\
\text { dikamentöse Therapie zur } \\
\text { Reduktion der erhöhten } \\
\text { Risikofaktoren }\end{array}$ \\
\hline \multirow[t]{2}{*}{$\begin{array}{l}20 \% \\
\text { oder: } \\
\text { bekannte Athero- } \\
\text { sklerose }\end{array}$} & $\begin{array}{l}5 \%-10 \% \\
\text { oder: } \\
\text { Cholesterin > } 8 \mathrm{mmol} / \mathrm{L} / \\
>320 \mathrm{mg} / \mathrm{dL} \\
\text { oder: } \\
\text { LDL-C > } 6 \mathrm{mmol} / \mathrm{L} />240 \mathrm{mg} / \mathrm{dL} \\
\text { oder: } \\
\text { Blutdruck }>180 / 110 \mathrm{~mm} \mathrm{Hg}\end{array}$ & hoch & $\begin{array}{l}<2,6 \mathrm{mmol} / \mathrm{L} \\
(<100 \mathrm{mg} / \mathrm{dL})\end{array}$ & $\begin{array}{l}\text { Wie oben plus in der Regel } \\
\text { intensive medikamentöse } \\
\text { Therapie zur Reduktion der } \\
\text { erhöhten Risikofaktoren }\end{array}$ \\
\hline & $\begin{array}{l}>10 \% \\
\text { oder: } \\
\text { bekannte Atherosklerose } \\
\text { oder: } \\
\text { Diabetes mellitus } \\
\text { oder: } \\
\text { chronische Niereninsuffizienz } \\
\text { mit GFR }<60 \mathrm{ml} / \mathrm{min}\end{array}$ & $\begin{array}{l}\text { sehr } \\
\text { hoch }\end{array}$ & $\begin{array}{l}<1,8 \mathrm{mmol} / \mathrm{L} \\
(<70 \mathrm{mg} / \mathrm{dL}),\end{array}$ & $\begin{array}{l}\text { Wie oben plus in der Regel } \\
\text { intensive medikamentöse } \\
\text { Therapie zur Reduktion der } \\
\text { erhöhten Risikofaktoren }\end{array}$ \\
\hline
\end{tabular}


Tab. 4.17 Definition des Metabolischen Syndroms (Alberti et al. 2009).

\begin{tabular}{lll}
\hline Messgrösse & Kategorische Cut-Offs & \\
\cline { 2 - 3 } & Männer & Frauen \\
\hline - Taillenumfang (gilt für Kaukasier, & $102 \mathrm{~cm}$ & $88 \mathrm{~cm}$ \\
andere Cut-Offs für andere Ethnizitäten) & (erhöhtes Risiko bei $94 \mathrm{~cm})$ & (erhöhtes Risiko bei $80 \mathrm{~cm})$ \\
- Triglyzeride (oder medikamentöse & $>1.7 \mathrm{mmol} / \mathrm{L}$ & $>1.7 \mathrm{mmol} / \mathrm{L}$ \\
$\begin{array}{l}\text { Behandlung einer Hypertriglyzeridämie) } \\
\text { - HDL-Cholesterin (oder medikamentöse }\end{array}$ & $(>150 \mathrm{mg} / \mathrm{dL})$ & $(>150 \mathrm{mg} / \mathrm{dL})$ \\
$\begin{array}{l}\text { Behandlung eines erniedrigten HDL- } \\
\text { Cholesterins) }\end{array}$ & $(<40 \mathrm{mg} / \mathrm{dL})$ & $<1.3 \mathrm{mmol} / \mathrm{L}$ \\
- Blutdruck (oder medikamentöse & & $(<50 \mathrm{mg} / \mathrm{dL})$ \\
anti-hypertensive Therapie) & Systol.: $>130 \mathrm{~mm} \mathrm{Hg}$ & Systol.: $>130 \mathrm{~mm} \mathrm{Hg}$ \\
- Glukose (oder medikamentöse & Diastol.: $>85 \mathrm{~mm} \mathrm{Hg}$ & Diastol.: $>85 \mathrm{~mm} \mathrm{Hg}$ \\
Behandlung einer Hyperglykämie) & $>5.5 \mathrm{mmol} / \mathrm{L}$ & $>5.5 \mathrm{mmol} / \mathrm{L}$ \\
\hline
\end{tabular}

Ein metabolisches Syndrom liegt vor, wenn drei von fünf Kriterien erfüllt sind.

fester Atherosklerose oder hohem Risiko wegen Diabetes mellitus oder dem Vorliegen multipler Risikofaktoren, die Absenkung des LDL-Cholesterins auf unter $2.6 \mathrm{mmol} / \mathrm{L}(100 \mathrm{mg} / \mathrm{dL})$ oder sogar auf unter $<1.8 \mathrm{mmol} / \mathrm{L}(<70 \mathrm{mg} / \mathrm{dL})$ empfohlen $(\triangleright$ Tab. 4.16, $\triangleright$ Abb. 4.10, 4.11) (The Task Force for the management of dyslipidaemias 2011). Für asymptomatische Patienten mit mittlerem oder niedrigem Risiko gelten höhere Zielwerte für LDLCholesterin $(\triangleright$ Tab. 4.16).

Die kausalen Beziehungen von HDL-Cholesterin und Triglyzeriden zur Arteriosklerose sind weniger klar als die des LDL-Cholesterins, auch weil niedriges HDL-Cholesterin und moderate Hypertriglyzeridämie häufig gemeinsam und mit anderen Komponenten des Metabolischen Syndroms (Übergewicht oder Adipositas, gestörte Glukosetoleranz oder manifester Diabetes mellitus Typ 2, Bluthochdruck) auftreten. Die statistisch unabhängige Assoziation vor allem des HDL-Cholesterins, weniger robust auch der Triglyzeride (Emerging Risk Factors Collaboration 2009) ist noch nicht beweisend. Bisherige Interventionsstudien haben keine Klarheit gebracht, auch weil die dabei eingesetzten Fibrate nur geringfügig die Konzentrationen des HDL-Cholesterins und Triglyzeride erhöhen bzw. senken. Insgesamt bewirkten Fibrate (allein oder in Kombination mit Statinen) keine signifikante Risikosenkung. In post hoc Analysen fand sich jedoch, dass Patienten mit gleichzeitig niedrigem HDL-Cholesterin $(<1 \mathrm{mmol} / \mathrm{L}$ oder $<38,4$ $\mathrm{mg} / \mathrm{dl})$ und erhöhten Triglyzeriden $(>2,3 \mathrm{mmol} / \mathrm{L}$ oder $>200$ mg/ dl) von der Fibrattherapie mit ge- ringeren Herzinfarktinzidenzen profitierten (Jun et al. 2010). Der klinische Nutzen der Nikotinsäure, welche die Plasmakonzentration des HDL-Cholesterins erhöht und die der Triglyzeride senkt, kann noch nicht abschliessend beurteilt werden, weil die Ergebnisse von randomisierten und kontrollierten Endpunktstudien zur Kombinationstherapie von Statinen und Nikotinsäure noch nicht vorliegen. Entsprechend gibt es für Triglyzeride und HDL-Cholesterin noch keine therapeutischen Zielwerte, sondern nur Grenzwerte für die Diagnose eines Metabolischen Syndroms und eines erhöhten kardiovaskulären Risikos ( $\triangleright$ Tab. 4.17) (Alberti et al. 2009).

\subsubsection{Differenzialdiagnose von Lipidstoff- wechselstörungen}

Lipidstoffwechselstörungen sind zumeist multifaktoriell bedingt, d. h. durch das Zusammentreffen genetischer Prädisposition und Umwelt- oder Lebensstilfaktoren. Insbesondere moderat ausgeprägte Dyslipidämien, die durch das Über- (LDLCholesterin, Triglyzeride) oder Unterschreiten (HDL-Cholesterin) der Ideal- und Zielwerte von Konsensus-Empfehlungen zur Prävention von kardiovaskulären Erkrankungen und Diabetes definiert sind ( $\triangleright$ Tab. 4.16 und 4.17), lassen sich im Einzelfall ätiologisch nicht eindeutig eruieren.

Insofern rechtfertigen nur ausgeprägte Dyslipidämien, bei denen z. B. die 90. oder 10. Perzentilen der Lipidstoffwechselparameter über- bzw. unterschritten werden $(\triangleright$ Tab. 4.18), den Aufwand einer 
eingehenden Differenzialdiagnostik und Ursachensuche.

Insbesondere die Suche nach monogenetischen Ursachen sollte wegen ihrer hohen Kosten und oft geringen therapeutischen Konsequenzen streng indiziert werden. Etliche monogenetische Dyslipidämien sind autosomal-kodominant vererbt, so dass durch Familienuntersuchungen die vertikalen Transmission des Lipid-Phänotyps nachgewiesen werden sollte, bevor aufwändige und teure genetische oder funktionelle Untersuchungen in Speziallaboratorien veranlasst werden. In $\triangleright$ Tab. 4.18 sind typische primäre und sekundäre Hyperlipidämien zusammengestellt.

Die Abb. 4.12 und 4.13 stellen die Diagnostischen Pfade für die Abklärung von Hypercholesterinämien, Hypertriglyzeridämien und Niedrig-HDLCholesterin-Dyslipidämie zusammen (Hersberger et al 2008).

\subsubsection{Porphyrien}

Porphyrien sind eine Gruppe von Stoffwechselstörungen der Häm-Biosynthese. Es handelt sich um genetische Erkrankungen mit Ausnahme der sporadischen Porphyria cutanea tarda (s. unten). Die meisten Porphyrien sind autosomal dominant vererbt, $\mathrm{d}$. h. bereits eine heterozygot vorliegende und funktionell relevante Mutation genügt für die Erkrankung. Da aber die Penetranz der Erkrankungen oft gering ist, ist dennoch häufig nur ein Individuum in einer Familie symptomatisch.

Die Porphyrien sind durch zwei Leitsymptome gekennzeichnet, die auch die Abklärungstrategien beinflussen: Neuroviszerale Attacken und Photodermatose. Die unten erwähnten Untersuchungsmethoden sind bei Minder und Schneider-Yin (2008) ausführlich beschrieben. Die vorgeschlagenen Abklärungsstrategien basieren auf konsensbasierten Empfehlungen und unsere Erfahrungen (www.porphyria-europe.org; Anderson et al. 2005; Schneider-Yin et al. 2009) ( Abb. 4.14 und 4.15).

\subsubsection{Neuroviszerale Attacken}

Das bei weitem häufigste Symptom ist die akute Abdominalsymptomatik. Sie tritt meistens mit weiteren typischen Symptomen wie Nausea, Erbrechen, Obstipation, Tachykardie, Hypertonie und Hyponatriämie. Peritonitische Zeichen fehlen und Entzündungszeichen wie Leukozytose oder Fieber sind selten. Bei Progredienz der Erkrankung kann es zu peripherer Neuropathie mit Lähmungen einschließlich der Atemmuskulatur, Parästhesien, epileptischen Anfällen und Coma mit möglicher Todesfolge kommen.

Mit dieser Symptomatik assoziierte Porphyrien werden heute als akute Porphyrien bezeichnet. Eine rasche Diagnose während einer akuten Phase durch Messung des quantitativen Porphobilinogens in einer Spontanurin-Probe standardisiert auf Kreatinin ermöglicht eine spezifische Behandlung mit Hämarginat (Normosang ${ }^{\circledR}$ ) und die Vermeidung von Porphyriea-auslösenden Medikamenten. Die qualitativen Nachweisverfahren von Porphobilinogen mit Ausnahme des Trace-Tests sind unspezifisch und ergeben daher viele falsch positive Resultate. Während eines akuten Porphyrieschubs bis mindestens eine Woche nach Symptombeginn ist Porphobilinogen mindestens 5-fach erhöht. Später und auch in einer Latenzphase kann bei einigen Patienten Porphobilinogen weiterhin massiv erhöht sein, während es sich bei anderen Patienten normalisiert.

Eine normale Porphobilinogen-Konzentration, die während einer typischen Schmerzattacke gemessen wurde, schließt die akute Porphyrie als Ursache der Schmerzen aus.

Drei unterschiedliche Erkrankungen können einer akuten Porphyrie zu Grunde liegen: Die akutintermittierende Porphyrie bedingt durch eine mutierte Porphobilinogen (PBG)-Deaminase, die Porphyria variegata bedingt durch eine mutierte Protoporphyrinogen-Oxidase und die hereditäre Koproporphyrie bedingt durch eine mutierte Koproporphyrinogen-Oxidase. Zusätzlich erwähnt werden soll der rezessive Defekt der Aminolävulinsäuredehydratase, der jedoch wegen seiner ausgesprochenen Seltenheit nicht in das Abklärungsschema einbezogen wurde. Die drei akuten Porphyrien können durch weitere biochemische Untersuchungen, PBG-Deaminase-Aktivität, Plasmafluoreszenz-Scan und Stuhlporphyrine, differenziert werden. Eine erniedrigte PBG-DeaminaseAktivität bei negativem Plasmafluoreszenzscan und normalen Stuhlporphyrinen ist typisch für die akut-intermittierende Porphyrie. Eine normale PBG-Deaminase-Aktivität, ein Peak im Plasmafluoreszenzscan bei $626 \mathrm{~nm}$, sowie erhöhte StuhlKonzentrationen von Koproporphyrin (KP) und Protoporphyrin (PPIX) sind typisch für die Porphyria variegata. Die hereditäre Koproporphyrie weist eine normale PBG-Deaminase-Aktivität auf, KP im Stuhl ist erhöht, PPIX im Stuhl normal und im Plas- 
Tab. 4.18 Differenzialdiagnosen zur Ursache von ausgeprägten Lipidstoffwechselstörungen; ABC = ATP Bindungskassettentransporter, $\mathrm{Apo}=$ Apolipoprotein, $\mathrm{ARH}=$ Autosomal rezessive Hypercholesterinämie, $\mathrm{LCAT}=$ Lezithin:Cholesterin:Acyltransferase, MTP = mikrosomales Transfer Protein, PCSK9 = Prokonvertase Subtilisin K9.

\begin{tabular}{|c|c|c|c|c|}
\hline & Cholesterin & Triglyzeride & LDL-Cholesterin & HDL-Cholesterin \\
\hline \multicolumn{5}{|l|}{ Hyperlipidämien } \\
\hline \multirow[t]{2}{*}{ 90. Perzentile (m/f) } & $7,5 / 7,5 \mathrm{mmol} / \mathrm{L}$ & $3,8 / 2,8 \mathrm{mmol} / \mathrm{L}$ & $5,0 / 5,0 \mathrm{mmol} / \mathrm{L}$ & $1,80 / 2,25 \mathrm{mmol} / \mathrm{L}$ \\
\hline & $(290 / 290 \mathrm{mg} / \mathrm{dL})$ & $(330 / 240 \mathrm{mg} / \mathrm{dL})$ & $(190 / 190 \mathrm{mg} / \mathrm{dL})$ & $(70 / 85 \mathrm{mg} / \mathrm{dL})$ \\
\hline \multirow[t]{3}{*}{$\begin{array}{l}\text { typische primäre } \\
\text { Ursachen }\end{array}$} & $\begin{array}{l}\text { Mutationen in Genen } \\
\text { für LDL-Rezeptor, ApoB, } \\
\text { PCSK9, ARH, ABCG5/8 } \\
\text { (Sitosterolämie) }\end{array}$ & $\begin{array}{l}\text { Chylomikronämie: } \\
\text { Mutationen in Genen } \\
\text { für Lipoproteinlipase, } \\
\text { ApoC-II, ApoA-V, } \\
\text { GPIHBP }\end{array}$ & $\begin{array}{l}\text { Mutationen in Genen } \\
\text { für LDL-Rezeptor, } \\
\text { ApoB, PCSK9, ARH, } \\
\text { ABCG5/8 (Sitoste- } \\
\text { rolämie) }\end{array}$ & $\begin{array}{l}\text { Mutationen in Genen } \\
\text { für CETP, hepatische } \\
\text { Lipase }\end{array}$ \\
\hline & \multicolumn{4}{|c|}{$\begin{array}{l}\text { Typ III Hyperlipidämie (ApoE2-Homozygotie, } \\
\text { andere Mutatione im ApoE-Gen) }\end{array}$} \\
\hline & \multicolumn{4}{|c|}{ Cholesterinester-Speicherkrankheit } \\
\hline \multirow{7}{*}{$\begin{array}{l}\text { typische sekundäre } \\
\text { Ursachen }\end{array}$} & Hypothyreose & Diabetes mellitus & Hypothyreose & \\
\hline & Cholestase & Niereninsuffizenz & Cholestase & \\
\hline & Anorexia nervosa & Hepatitis & Anorexia nervosa & \\
\hline & & Entzündungen & & \\
\hline & & Schwangerschaft & & \\
\hline & Neprotisches Syndrom & & & \\
\hline & Cushing-Syndrom & & & \\
\hline \multirow{6}{*}{$\begin{array}{l}\text { typische Medika- } \\
\text { menten- oder } \\
\text { Nahrungsmittel- } \\
\text { wirkungen }\end{array}$} & Gestagene & Östrogene & Gestagene & Östrogene \\
\hline & HIV-Protease-Inhibitoren & Diuretika & HIV-Protease & Antikonvulsiva \\
\hline & Immunsuppressiva & Neuroleptika & Inhibitoren & \\
\hline & & Glukokortikoide & Immunsuppressiva & \\
\hline & & Retinoide & & \\
\hline & & Alkohol & & \\
\hline \multicolumn{5}{|l|}{ Hypolipidämien } \\
\hline \multirow[t]{2}{*}{ 10. Perzentile (m/f) } & $4,7 / 4,7 \mathrm{mmol} / \mathrm{L}$ & $0,7 / 0,9 \mathrm{mmol} / \mathrm{L}$ & $2,3 / 2,3 \mathrm{mmol} / \mathrm{L}$ & $0,90 / 1,15 \mathrm{mmol} / \mathrm{L}$ \\
\hline & $180 / 180$ mg/dL & $80 / 60 \mathrm{mg} / \mathrm{dl}$ & $90 / 90 \mathrm{mg} / \mathrm{dL}$ & $35 / 45 \mathrm{mg} / \mathrm{dL}$ \\
\hline $\begin{array}{l}\text { typische primäre } \\
\text { Ursachen }\end{array}$ & $\begin{array}{l}\text { A/Hypobetalipopro- } \\
\text { teinämie (Mutationen in } \\
\text { ApoB, MTP) }\end{array}$ & & $\begin{array}{l}\text { A/Hypobetalipopro- } \\
\text { teinämie (Mutationen } \\
\text { in ApoB, MTP) }\end{array}$ & $\begin{array}{l}\text { Mutationen in Genen } \\
\text { für ApoA-I, LCAT, } \\
\text { ABCA1 (Tangier Di- } \\
\text { sease) }\end{array}$ \\
\hline $\begin{array}{l}\text { typische sekun- } \\
\text { däre Ursachen }\end{array}$ & $\begin{array}{l}\text { Leberversagen, Sepsis, } \\
\text { katabole Zustände }\end{array}$ & Leberversagen & $\begin{array}{l}\text { Leberversagen, } \\
\text { Sepsis, katabole } \\
\text { Zustände }\end{array}$ & $\begin{array}{l}\text { Diabetes (Metaboli- } \\
\text { sches Syndrom), Le- } \\
\text { berversagen, Rechts- } \\
\text { herz-versagen, Akute } \\
\text { Entzündungen, Malig- } \\
\text { nome (hämato-onkolo- } \\
\text { gische Erkrankungen), }\end{array}$ \\
\hline $\begin{array}{l}\text { typische Medika- } \\
\text { menten- oder } \\
\text { Nahrungsmittel- } \\
\text { wirkungen }\end{array}$ & & & & $\begin{array}{l}\text { Androgene, Anabo- } \\
\text { lika, einige beta- } \\
\text { Blocker }\end{array}$ \\
\hline
\end{tabular}




\section{Abklärung der Hypercholesterin- und Hypertriglyzeridämie}

\section{Eingangs- untersuchung/ Zufallsbefund}
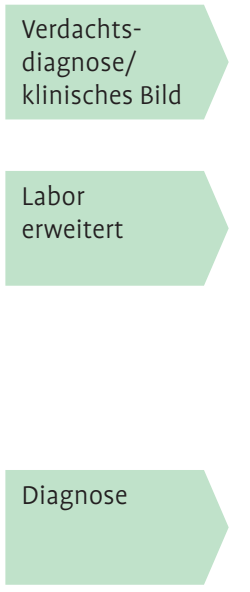

Abklärung
weiterer
Ursachen

\section{Lipidstatus}

LDL/Gesamt-Cholesterin > 90. Perzentile

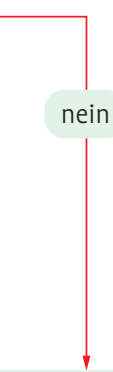

gemischte

Hyperlipidämie

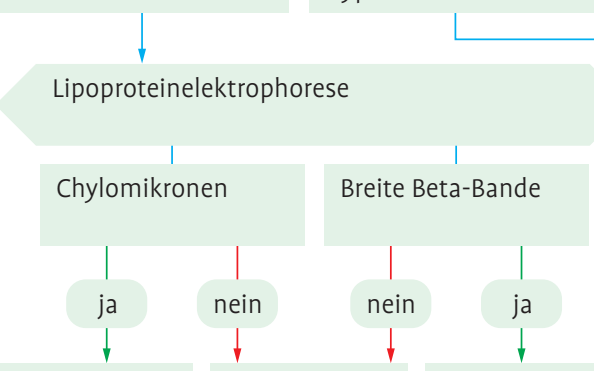

HLP Typ V isolierte/

vorherrschende

Hypercholesterinämie keine oder moderate

Hypercholesterinämie

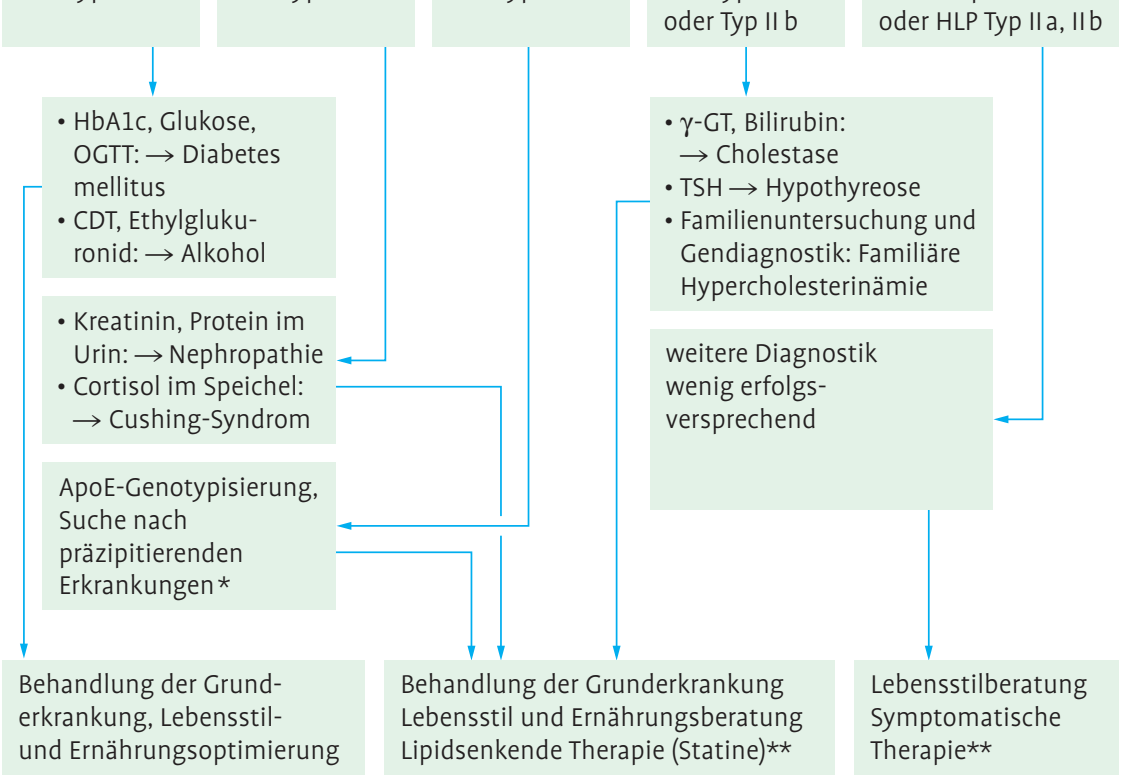

Abb.4.12 Flussdiagramm zur Differenzialdiagnostik der Hypercholesterinämie; *: Typische die Typ III Hyperlipoproteinämie präzipitierende Krankheiten bei ApoE2Homozygotie sind Diabetes mellitus, Hypothyreose, Hämochromatose; **: Vorgehen wie in Abbildungen
4.7, 4.8 oder 4.9 und 4.16 beschrieben; Perzentilgrenzen siehe Tabelle 4.18; HLP = Hyperlipoproteinämie (Typenbezeichnung nach Fredrickson), ApoE = Apolipoprotein $\mathrm{E}, \mathrm{CDT}=$ carbohydrat-defizientes Transferrin, $\mathrm{TSH}=$ thereoideastimulierendes Hormon. 
Abklärung der Hypertriglyzeridämie und der Hypoalphalipoproteinämie

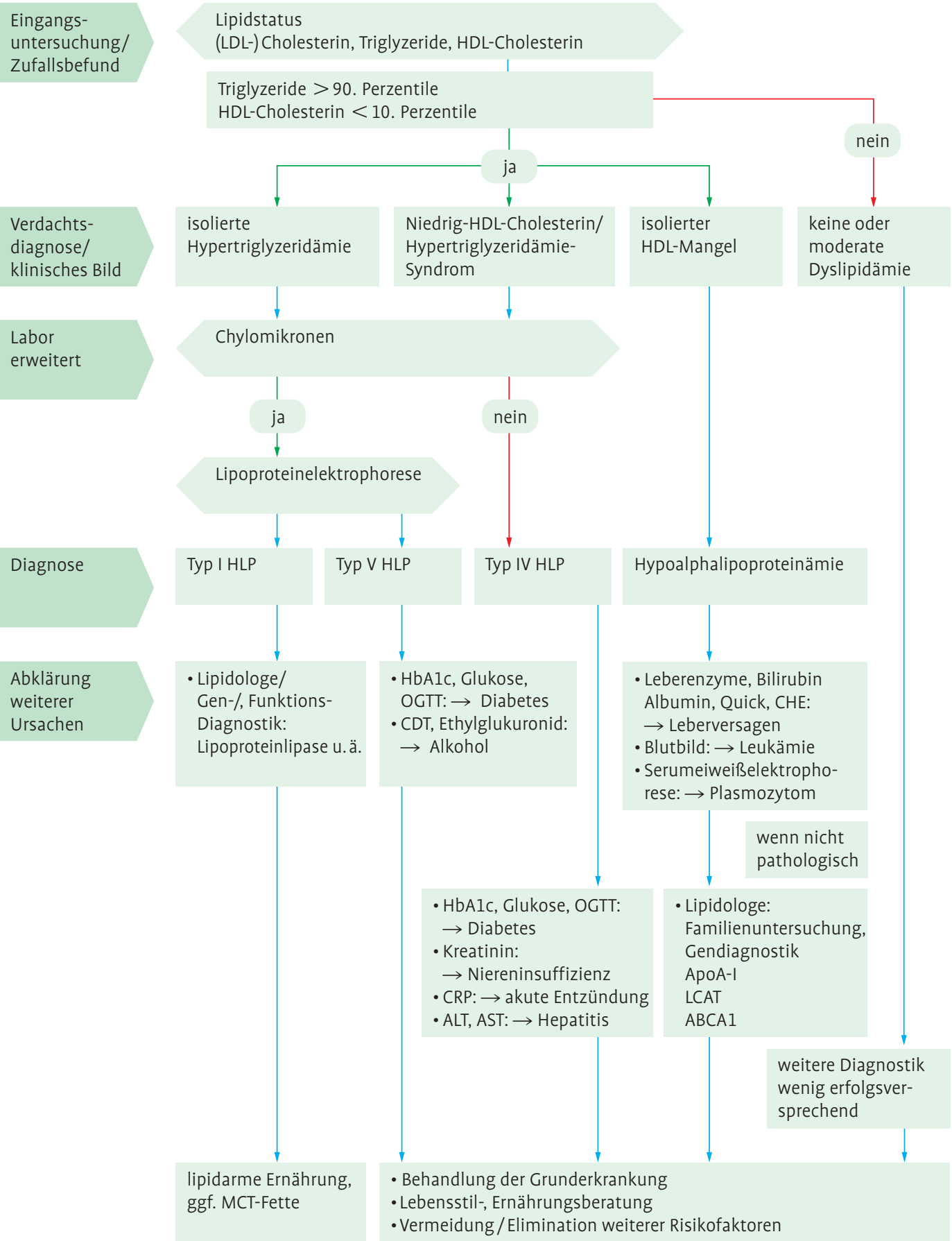

Abb.4.13 Flussdiagramm zur Differenzialdiagnostik der Hypertriglyzeridämie und des niedrigen HDL-Cholesterins; Perzentilgrenzen siehe Tabelle 4.18; HLP = Hyperlipoproteinämie (Typenbezeichnung nach Fredrickson), CDT = carbohydrat-defizientes Transferrin, OGTT = ora- ler Glukosetoleranztest, $\mathrm{CHE}=$ Cholinesterase, $\mathrm{CRP}=$ C-reaktives Protein, ALT = Alanin-Aminotransferase, AST $=$ Aspartat-Aminotransferase, ApoA-I = Apolipoprotein A-I, ABCA1 = ATP-Bindungskassetten-Transporter A1, LCAT = Lezithin:Cholesterin-Acyltransferase. 
mafluoreszenzscan zeigt sich häufig ein Peak bei $620 \mathrm{~nm}$. Diese Diagnosen werden anschliessend verifiziert durch eine Untersuchung der entsprechenden Gene, Hydroxymethylbilansynthase (HMBS) für die akut-intermittierende Porphyrie,
Protoporphyrinogen-Oxidase (PPOX) für die Porphyria variegata und Koproporphyrinogen-Oxidase (CPOX) für die hereditäre Koproporphyrie.

Abklärung in Latenzphase: Sollte eine Abklärung bei einem Patienten notwendig werden, bei dem

\section{Porphyrieverdacht: Abklärung akute Abdominalkoliken bei symptomatischem Patient}

Erstuntersuchung

\section{Porphobilinogen/Kreatinin \\ (Kreatininkonzentration mindestens $4 \mathrm{mmol} / \mathrm{L}$ )}

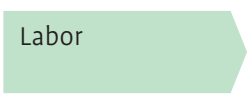

erste Diagnose

Labor

biochemische Differenzierung

\section{Labor}

molekulargenetische Diagnose

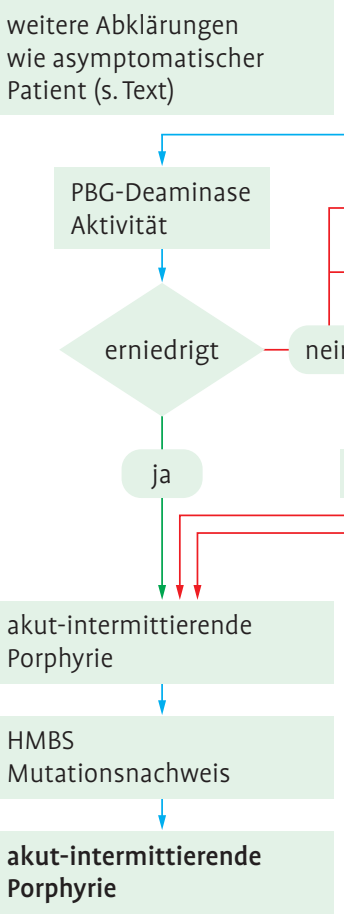

Porphyrie

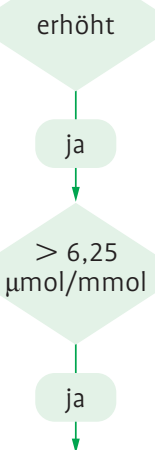

akute Porphyrie
Porphyrie ausgeschlossen

Abb. 4.14 Flussdiagramm zur Differenzialdiagnostik der mit Abdominalkoliken einhergehenden Porphyrien; PBG: Porphobilinogen, KP: Koproporphyrin, PPIX: Pro-

toporphyrin, HMBJ: Hydroxymethylbilan-Synthase, PPOX: Protoporphyrinogen-Oxidase, CPOX: Koproporphyrinogen-Oxidase. 
die Symptomatik länger als eine Woche zurückliegt oder ist das Porphobilinogen weniger als 5-fach erhöht, so empfehlen wir neben der Bestimmung von Porpobilinogen, die Messung der PBG-DeaminaseAktivität, des Plasmafluoreszenzscans und der Stuhlporphyrine. Die Kombination dieser vier Tests hilft mit einer Sensitivität von über $90 \%$ die Diagnosestellung einer akuten Porphyrie.

Bleibt danach weiterhin eine Unklarheit bestehen, so sollte eine erneute Schmerzattacke abgewartet werden und dann entsprechend dem Schema für symptomatische Patienten vorgegangen werden.

Familienabklärung: Bei allen akuten Porphyrien empfehlen wir die Blutsverwandten des Indexpa- tienten auf Porphyrie abzuklären, um bei Betroffenen vorbeugende Massnahmen zu ergreifen. Blutsverwandte haben ein $50 \%$-Risiko, ebenfalls die Porphyrie geerbt zu haben. Am zuverlässigsten erfolgt diese Abklärung über die genetische Suche nach der beim Index-Patienten vorliegenden Mutation.

\subsubsection{Photodermatosen}

Diese Licht-ausgelösten Hauterkrankungen sind begrenzt auf belichtete Hautstellen. Am meisten betroffen sind Handrücken und Gesicht. Dabei sind zwei Krankheitsbilder zu unterscheiden, die bekanntere blasenbildende ( $\downarrow$ Abb. 4.16 a) und die

\section{Abklärung Photodermatose}

Erstuntersuchung

erste Diagnose

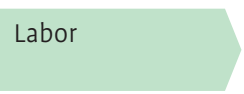

biochemische Differenzierung

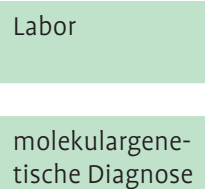

Plasmafluoreszenzscan

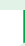

Peak

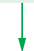

kutane Porphyrie kutane Porphyrie ausgeschlossen

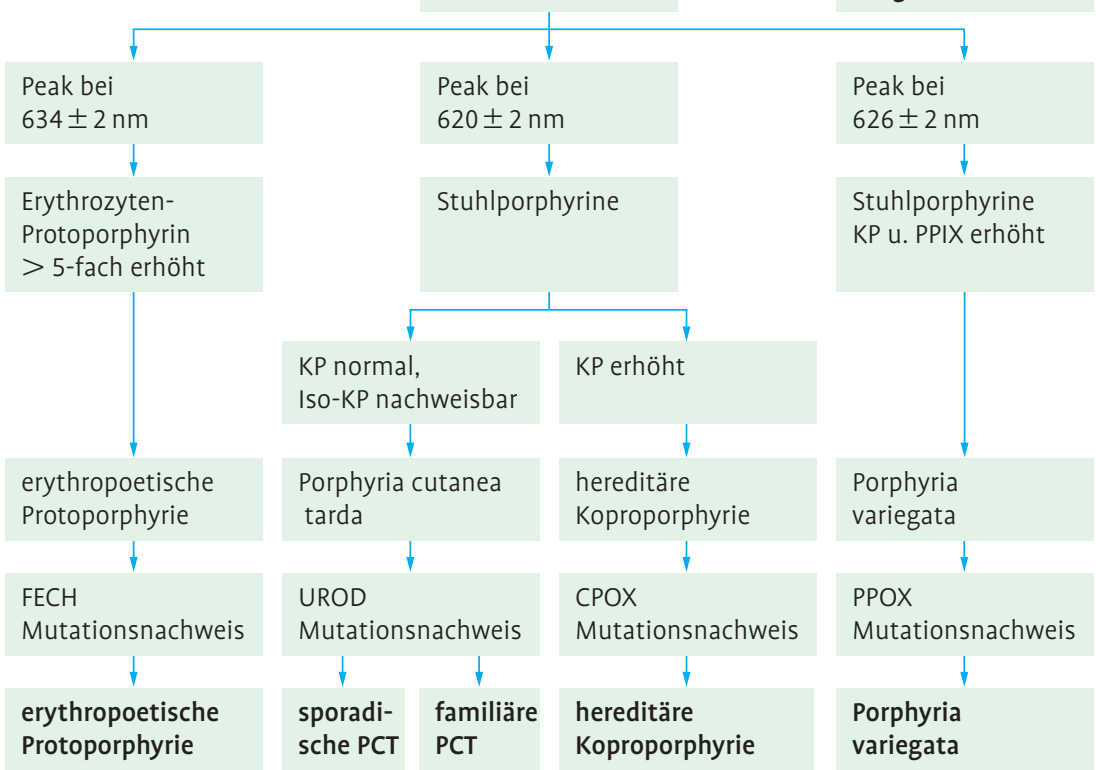

Abb.4.15 Flussdiagramm zur Differenzialdiagnostik der mit Photodermatosen einhergehenden Porphyrien. 


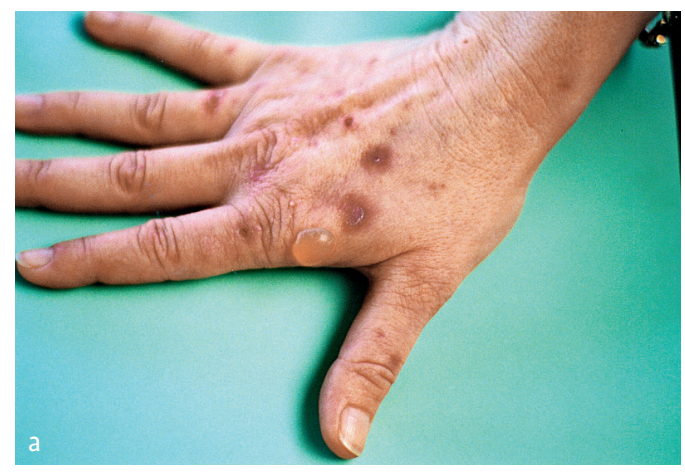

Abb.4.16a, b Photodermatosen bei Porphyrie.

akute schmerzhafte Hautkrankheit ( Abb. 4.16b). Während die erste sich vor allem im Erwachsenenalter manifestiert, tritt die zweite typischerweise im Kindesalter auf.

Ein einfacher Screeningtest für eine kutane Porphyrie ist das Plasmafluoreszenzscan. Dabei wird ein im Plasma vorhandenes Porphyrin bei einer Wellenlänge von $410 \mathrm{~nm}$ angeregt und das Emissionsspektrum zwischen 500 und $700 \mathrm{~nm}$ gemessen. Das Vorliegen eines Emissions-Peaks zwischen 615 und $636 \mathrm{~nm}$ erlaubt die Verdachtsdiagnose Porphyrie, das Fehlen dieses Peaks schliesst eine kutane Porphyrie mit hoher Wahrscheinlichkeit aus. Die Lage des Peak-Maximums lässt bereits eine gewisse Differenzierung der Porphyrie zu: Das Maximum bei $626 \mathrm{~nm}$ ist charakteristisch für die Porphyria variegata, jenes bei $635 \mathrm{~nm}$ für die erythropoietische Protoporphyrie. Einzig das PeakMaximum um $620 \mathrm{~nm}$ kann durch verschiedene Porphyrien verursacht sein. Die wichtigsten davon sind die Porphyria cutanea tarda und die hereditäre Koproporphyrie. Diese beiden Porphyrien lassen sich durch die Untersuchung der Stuhlporphyrine differenzieren: Die erste ist durch das Vorliegen von Isokoproporphyrin gekennzeichnet, die zweite durch erhöhtes Koproporphyrin. Weitere Porphyrien mit einem Peak-Maximum bei $620 \mathrm{~nm}$, die wegen ihrer Seltenheit nicht in das Abklärungsschema aufgenommen wurden, sind die kongenitale erythropoetische Porphyrie und die akut intermittierende Porphyrie mit der Komplikation einer dialysepflichtigen Niereninsuffizienz. Die kongenitale erythropoetische Porphyrie ist charakterisiert durch das dominante Auftreten von I-Isomere in allen Körperflüssigkeiten. Die akut intermittierende Porphyrie mit Niereninsuffizienz lässt sich durch normale Stuhlporphyrine er-

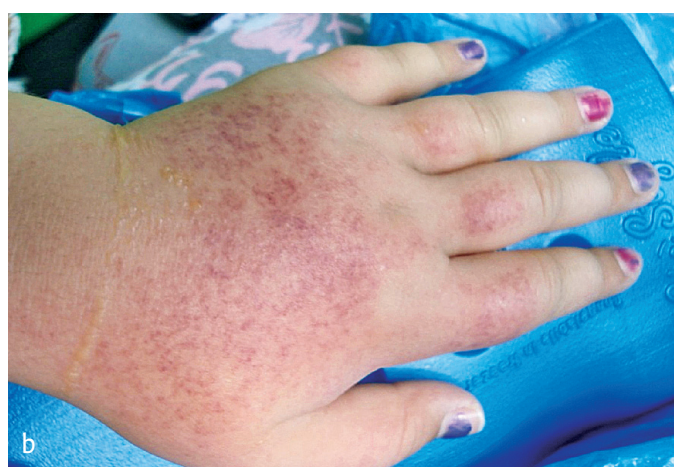

kennen. Die bei dieser Porphyrie normalerweise erniedrigte PBG-Deaminase-Aktivität kann wegen der komplizierenden hämatologischen Situation falsch normal sein. Die Porphyria variegata lässt sich ebenfalls durch die Messung der Stuhlporphyrine bestätigen, die erhöhtes Koproporphyrin und erhöhtes Protoporphyrin zeigen. Die erythropoetische Protoporphyrie dagegen weist ein mindestens 5-fach erhöhtes Erythrozytenprotoporphyrin aus.

Die genetische Untersuchung bestätigt die Diagnosen. Bei der Porphyria cutanea tarda lässt sich die familiäre Form von der sporadischen unterscheiden. Der Typus der Mutation der erythropoetischen Protoporphyrie hat einen Einfluss auf das Risiko für eine komplizierende Protoporphyrin-bedingte Lebererkrankung. Bei den beiden akuten Porphyrien, hereditäre Koproporphyrie und Porphyria variegata, empfiehlt sich die Mutationssuche beim Indexpatienten, um die empfohlene Familienabklärung zu erleichtern.

\section{Literatur}

Alberti KG, Eckel RH, Grundy SM, Zimmet PZ, Cleeman JI, Donato KA, Fruchart JC, James WP, Loria CM, Smith SC Jr; International Diabetes Federation Task Force on Epidemiology and Prevention; Hational Heart, Lung, and Blood Institute; American Heart Association; World Heart Federation; International Atherosclerosis Society; International Association for the Study of Obesity. Harmonizing the metabolic syndrome: a joint interim statement of the International Diabetes Federation Task Force on Epidemiology and Prevention; National Heart, Lung, and Blood Institute; American Heart Association; World Heart Federation; International Atherosclerosis Society; and International Association for the 
Study of Obesity. Circulation. 2009 Oct 20;

120(16): 1640-5.

American Diabetes Association. Report of the Expert Committee on the Diagnosis and Classification of Diabetes Mellitus. Diabetes Care 1997; 20: 11831197.

American Diabetes Association. Standards of medical care in diabetes-2010. Diabetes Care. 2010 Jan; 33 Suppl 1: S11-61. Erratum in: Diabetes Care. 2010 Mar; 33(3): 692.

Anderson KE, Bloomer JR, Bonkovsky HL, Kushner JP, Pierach CA, Pimstone NR, Desnick RJ. Recommendations for the diagnosis and treatment of the acute porphyrias. Ann Intern Med. 2005 15; 142 (6): 439-50.

Assmann G, Cullen P, Fruchart JC, Greten H, Naruszewicz M, Olsson A, Paoletti R, Riesen W, Stoll M, Tikkanen $M$, von Eckardstein $A$; for the International Task Force for Prevention of Coronary Heart Disease. Implications of emerging risk factors for therapeutic intervention. Nutr Metab Cardiovasc Dis. 2005 Oct; 15(5): 373-81.

Blankenberg S, Zeller T, Saarela O, Havulinna AS, Kee F, Tunstall-Pedoe H, Kuulasmaa K, Yarnell J, Schnabel RB, Wild PS, Münzel TF, Lackner KJ, Tiret L, Evans A, Salomaa V; MORGAM Project. Contribution of 30 biomarkers to 10-year cardiovascular risk estimation in 2 population cohorts: the MONICA, risk, genetics, archiving, and monograph (MORGAM) biomarker project. Circulation. 2010 Jun 8; 121(22): 2388-97

Brown A, Reynolds LR, Bruemmer D. Intensive glycemic control and cardiovascular disease: an update. Nat Rev Cardiol. 2010 Jul; 7(7): 369-75

Castorino KN, Jovonivic L. Pregnancy and diabetes management: Advances and Controversies. Clin. Chem. 2011, 57(2): 221-30

Cholesterol Treatment Trialists' (CTT) Collaborators, Kearney PM, Blackwell L, Collins R, Keech A, Simes J, Peto R, Armitage J, Baigent C. Efficacy of cholesterol-lowering therapy in 18,686 people with diabetes in 14 randomised trials of statins: a meta-analysis. Lancet. 2008 Jan 12; 371(9607): 117-25.

Cholesterol Treatment Trialists' (CTT) Collaboration, Baigent C, Blackwell L, Emberson J, Holland LE, Reith C, Bhala N, Peto R, Barnes EH, Keech A, Simes J, Collins R. Efficacy and safety of more intensive lowering of LDL cholesterol: a meta-analysis of data from 170,000 participants in 26 randomised trials. Lancet. 2010 Nov 13; 376(9753): 1670-81

Currie CJ, Peters JR, Tynan A, Evans M, Heine RJ, Bracco OL, Zagar T, Poole CD. Survival as a function of $\mathrm{HbA}(1 \mathrm{c})$ in people with type 2 diabetes: a retrospective cohort study. Lancet. 2010 Feb 6; 375(9713): 481-9.

Ekelund M, Shaat N, Almgren P, Groop L, Berntorp K. Prediction of postpartum diabetes in women with gestational diabetes mellitus. Diabetologia. 2010 Mar; 53(3): 452-7

Emerging Risk Factors Collaboration, Di Angelantonio E, Sarwar N, Perry P, Kaptoge S, Ray KK, Thompson A, Wood AM, Lewington S, Sattar N, Packard CJ, Collins R, Thompson SG, Danesh J. Major lipids, apolipoproteins, and risk of vascular disease. JAMA. 2009 Nov 11; 302(18): 1993-2000

Fioretto P, Dodson PM, Ziegler D, Rosenson RS. Residual microvascular risk in diabetes: unmet needs and future directions. Nat Rev Endocrinol. 2010 Jan; 6(1): 19-25.

Hersberger M, Rohrer L, von Eckardstein A (2008). Lipoproteins In: Blau N, Duran M, Gibson KM, editors. Physicians Guide to the Methods in Biochemical Genetics, Heidelberg Springer Verlag 2008

International Association of Diabetes and Pregnancy Study Groups Consensus Panel, Metzger BE, Gabbe SG, Persson B, Buchanan TA, Catalano PA, Damm P, Dyer AR, Leiva A, Hod M, Kitzmiler JL, Lowe LP, McIntyre HD, Oats JJ, Omori Y, Schmidt MI. International association of diabetes and pregnancy study groups recommendations on the diagnosis and classification of hyperglycemia in pregnancy. Diabetes Care. 2010 Mar; 33(3): 676-82.

International Expert Committee. International Expert Committee report on the role of the A1c assay in the diagnosis of diabetes. Diabetes Care 2009; 32: 1327-1334

Jun M, Foote C, Lv J, Neal B, Patel A, Nicholls SJ, Grobbee DE, Cass A, Chalmers J, Perkovic V. Effects of fibrates on cardiovascular outcomes: a systematic review and meta-analysis. Lancet. 2010 May 29; 375(9729): 1875-84

Keil U, Fitzgerald AP, Gohlke H, Wellmann J, Hense HW. Risikoabschätzung tödlicher Herz-Kreislauf-Erkrankungen: Die neuen SCORE-Deutschland-Tabellen für die Primärprävention. Deutsches Ärzteblatt 102, A-1808-A1812. 2005

Kerner W, Brückel J. Definition, Klassifikation und Diagnostik. Diabetologie 2010; 5: S 109-S 112

Lenters-Westra E, Slingerland RJ. Six of eight hemoglobin A1c point-of-care instruments do not meet the general accepted analytical performance criteria. Clin Chem. 2010 Jan; 56(1): 44-52.

Matthaei S, Bierwirth R, Fritsche A, Gallwitz B, Häring $\mathrm{H}-\mathrm{U}$, Joost $\mathrm{H}-\mathrm{G}$, Kellerer M, Kloos C, Kunt T, Nauck M, Schernthaner G, Siegel E, Thienel F. Behandlung des Diabetes mellitus Typ 2. Diabetologie 2010; 5: S $127-S 132$

Minder El, Schneider X. Porphyrins, Porphobilinogen and d-Aminolevulinic Acid. In: Blau N, Duran M, Gibson KM, editors. Physicians Guide to the Methods in Biochemical Genetics, Heidelberg Springer Verlag 2008: 751-780.

National Diabetes Data Group. Classification and diagnosis of diabetes mellitus and other categories of 
glucose intolerance. National Diabetes Data Group. Diabetes 1979; 28: 1039-1057.

Nordestgaard BG, Chapman MJ, Ray K, Borén J, Andreotti $F$, Watts GF, Ginsberg $H$, Amarenco $\mathrm{P}$, Catapano A, Descamps OS, Fisher E, Kovanen PT, Kuivenhoven JA, Lesnik P, Masana L, Reiner Z, Taskinen MR, Tokgözoglu L, Tybjærg-Hansen A; European Atherosclerosis Society Consensus Panel Lipoprotein(a) as a cardiovascular risk factor: current status. Eur Heart J. 2010 Dec; 31(23): 2844-53.

Rathmann W, Kowall B, Tamayo T, Giani G, Holle R, Thorand B, Heier M, Huth C, Meisinger C Hemoglobin A1c and glucose criteria identify different subjects as having type 2 diabetes in middle-aged and older populations: The KORA S4/F4 Study. Ann Med. 2010 Nov 22. (Epub ahead of print)

Schulze MB, Hoffmann K, Boeing H, Linseisen J, Rohrmann S, Möhlig M, Pfeiffer AF, Spranger J, Thamer C, Häring HU, Fritsche A, Joost HG. An accurate risk score based on anthropometric, dietary, and lifestyle factors to predict the development of type 2 diabetes. Diabetes Care 30, 510-5, 2007

The Task Force for the management of dyslipidaemias. ESC/EAS Guidelines for the management of dyslipidaemias. European Heart Journal (2011) 32, 17691818

von Eckardstein A, Schulte H, Assmann G. Vergleich internationaler Konsensus-Empfehlungen zur Erkennung des präsymptomatischen Hochrisikopatienten für den Herzinfarkt in Deutschland. Z Kardiol. 2005 Jan; 94(1): 52-60.

World Health Organization. Definition, diagnosis and classification of diabetes mellitus and its complications: report of a WHO consultation. Part 1: diagnosis and classification of diabetes mellitus 1999 World Health Organization Geneva

\subsection{Leber- und Pankreaserkrankungen}

Max G. Bachem, Thomas Seufferlein, Marco Siech

\subsubsection{Hepatobiliäre Erkrankungen} (Abb. 4.17)

Hepatobiliäre Erkrankungen kann man entsprechend ihrer Ursache, ihrem Erscheinungsbild und ihrer Laborkonstellation folgendermaßen einteilen:

- Erkrankungen, die mit einer hepatozellulären Schädigung einhergehen

- cholestatische Erkrankungen

- gemischt-hepatozelluläre/cholestatische Erkrankungen

- Erkrankungen mit reduzierter Syntheseleistungund Entgiftungsfunktion
Klinisch können sich hepatobiliäre Erkrankungen durch abdominelle Schmerzen im im Bereich des rechten oberen Quadranten äußern, wobei ein Dauerschmerz oder kolikartige Schmerzen bestehen können. Zusätzlich kann ein Ikterus vorhanden sein. In vielen Fällen wird die hepatobiliäre Erkrankung jedoch als Zufallsbefund durch erhöhte Transaminasen oder gamma-GT festgestellt.

\section{Anamnese}

Bei Verdacht auf eine hepatobiliäre Erkrankung sollte bei der Anamnese insbesondere nach Zeichen einer Lebererkrankung gefragt werden. Müdigkeit, die sich äußert durch erhöhtes Schlafbedürfnis, Lethargie, Schwäche, Krankheitsgefühl ist das häufigste Symptom bei Lebererkrankungen.

Bei Schmerzen im rechten Oberbauch, interessieren die Art und Dauer der Schmerzen. Weiterhin sollte erfragt werden, ob Juckreiz vorhanden ist, ob sich die Stuhl- und Urinfarbe verändert hat, wie ausgeprägt der Alkoholkonsum ist, ob Kontakt zu Personen mit Gelbsucht bestand, ob Gallensteine bekannt sind, ob es in letzter Zeit zu einem Gewichtsverlust gekommen ist, ob das Vorhandensein von entzündlichen Darmerkrankungen bekannt ist, ob eine Autoimmunerkrankung bekannt ist, ob kürzlich ein Auslandsaufenthalt bestanden hat, welche Medikamente eingenommen werden und ob parenteral Drogen konsumiert werden. Im Hinblick auf sexuell übertragbare Virushepatitiden sollte auch nach den sexuellen Aktivitäten gefragt werden. Bei Personen, die im medizinischen Bereich tätig sind, sollte eine Infektion durch Nadelstichverletzung ausgeschlossen werden.

\section{Körperliche Untersuchung}

Häufig ist die körperliche Untersuchung bei Patienten mit hepatobiliären Erkrankungen nicht besonders zielführend. Akute Affektionen, schwere Verlaufsformen und fortgeschrittene Lebererkrankungen können jedoch durch das Vorhandensein eines Ikterus, durch abdominelle Schmerzen im Bereich der Leber, eine Leber- oder Milzvergrößerung, evtl. ein Aszites oder pathologische Umgehungskreisläufe oder einen "foetor hepaticus" erkannt werden. Auch auf Zeichen eines chronischen Alkoholabusus sollte bei der körperlichen Untersuchung besonders geachtet werden. Eine evtl. vorhandene Hyperpigmentierung könnte auf eine 